\title{
FABRICATION OF SIEVED-MICROWELLS FOR SINGLE PARTICLE CAPTURE
}

\author{
by \\ Lauren Romita \\ B.Eng, Ryerson University, Toronto, 2017
}

\author{
A thesis \\ presented to Ryerson University \\ in partial fulfillment of the \\ requirements for the degree of \\ Master of Applied Science \\ in the program of \\ Chemical Engineering
}

Toronto, Ontario, Canada, 2020

(C) Lauren Romita, 2020 


\section{Author's Declaration}

I hereby declare that I am the sole author of this thesis. This is a true copy of the thesis, including any required final revisions, as accepted by my examiners.

I authorize Ryerson University to lend this thesis to other institutions or individuals for the purpose of scholarly research.

I further authorize Ryerson University to reproduce this thesis by photocopying or by other means, in total or in part, at the request of other institutions or individuals for the purpose of scholarly research.

I understand that my thesis may be made electronically available to the public. 


\section{Abstract}

Fabrication of Sieved-Microwells for Single Particle Capture, MASc, 2020, Lauren Romita, Chemical Engineering, Ryerson University

The use of microwells is popular for a wide range of applications due to its' ease of use. However, the seeding of conventional microwells, which are closed at the bottom, is restricted to gravitational sedimentation for cell or particle deposition and therefore require lengthy settling times to maximize well occupancy. Microfluidics has accelerated cell or particle capture through flow but is mostly limited to gravitationally-driven settling for capture into the wells. An alternative approach to conventional closed-microwells, sieved microwells supersedes reliance on gravity by using hydrodynamic forces through the open pores at the bottom of the microwells to draw targets into the wells. The aim of this thesis is to develop a rapid and high-throughput fabrication method for sieved microwells and integrate the microwells into a double-layered microfluidic device to enable crossflow trapping. The resulting device achieves an $87 \%$ well occupancy in under 10 seconds. 


\section{Acknowledgements}

I would like to express my sincere thanks to my supervisor, Dr. Dae Kun Hwang, for his continuous support and guidance. His expertise and encouragement, in addition to his patience and enthusiasm, were instrumental in completing this research.

I would like to thank Daniel Foster, Seungyoon Han and Shyan Thompson for their assistance and advice in completing this research. In addition, to thanking all my lab members for their support and positivity which made this such a gratifying experience.

Finally, I wish to thank my family for their support and encouragement throughout my studies. I could not have done this without you. 


\section{Table of Contents}

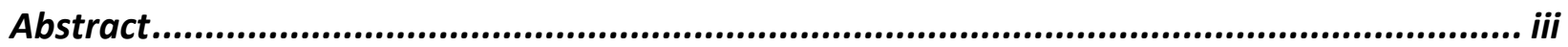

List of Tables................................................................................................. vii

List of Figures................................................................................................... viii

List of Abbreviations .........................................................................................

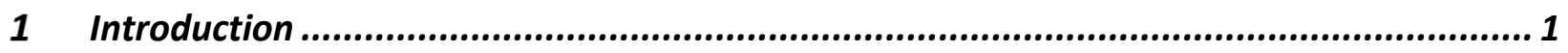

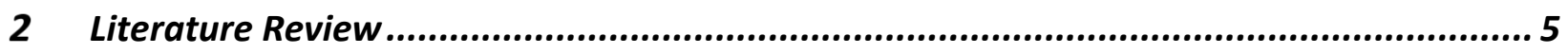

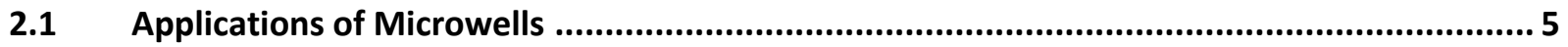

2.1.1 Specific Applications of Microfluidic-Integrated Microwells ...........................................................

2.2 Design Considerations for Microwells .......................................................................... 6

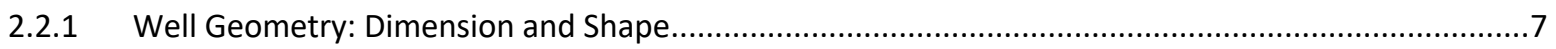

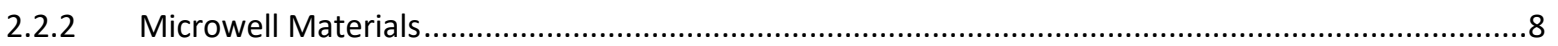

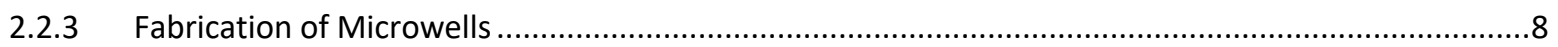

2.3 Trapping Cells/Particles into Microwells .......................................................................... 11

2.3.1 Trapping Targets in Static Microwells................................................................................... 11

2.3.2 Trapping Targets in Microfluidic Integrated Microwell Systems .............................................14

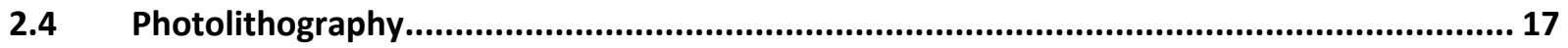

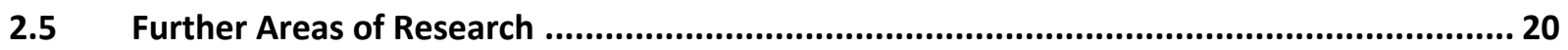

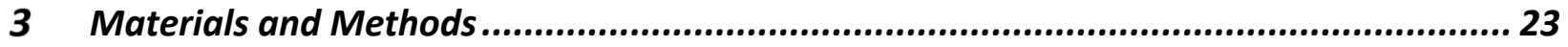

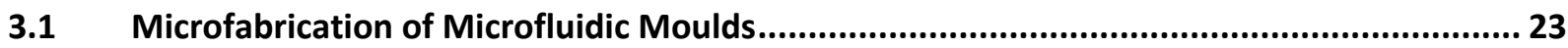

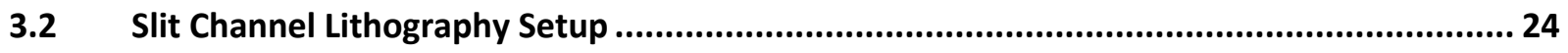

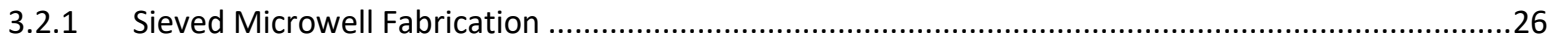




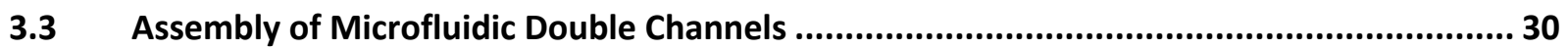

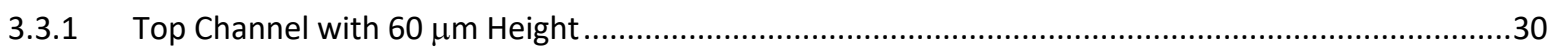

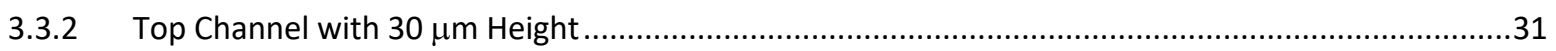

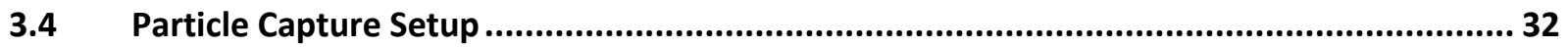

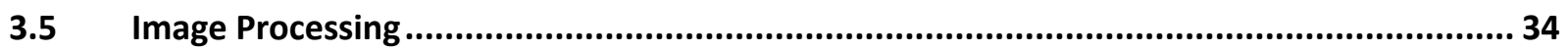

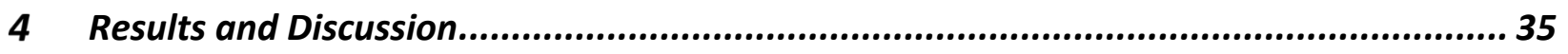

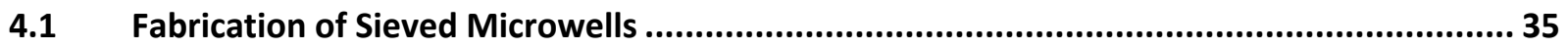

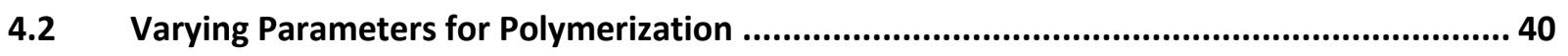

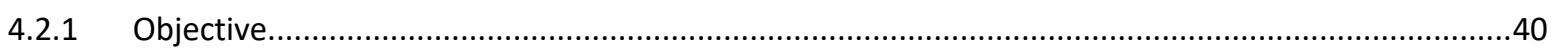

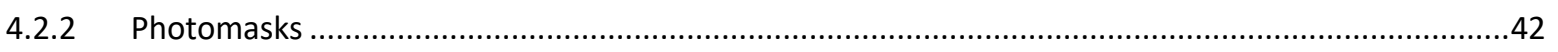

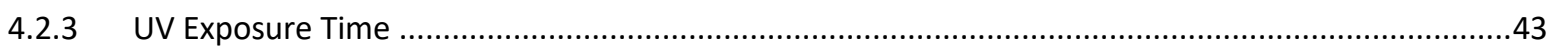

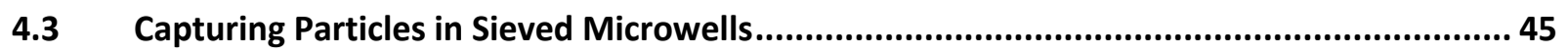

4.3.1 Microwell and Particle Size Selection .......................................................................................

4.3.2 Assembling and Using a $60 \mu \mathrm{m}$ Tall Top Channel ......................................................................48

4.3.3 Assembling and Using a $30 \mu \mathrm{m}$ tall Top Channel......................................................................51

5 Conclusions and Recommendations......................................................... 55

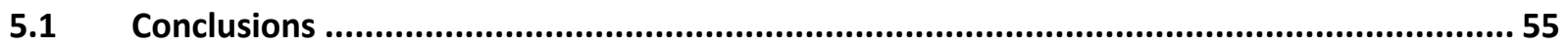

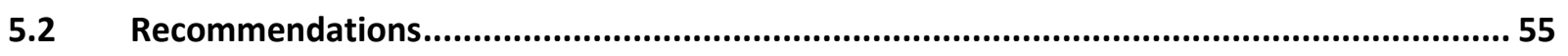

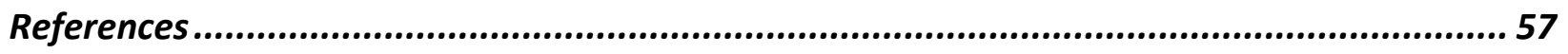




\section{List of Tables}

Table 1. Summary of Static Particle Capture Methods .......................................................12

Table 2. Summary of Trapping in Microfluidic Devices ....................................................15

Table 3. Operating Conditions for Slit Channel Lithography ...............................................28 


\section{List of Figures}

Figure 1. Particles being captured in porous microfluidic device .........................................4

Figure 2. Examples microwells geometries and fabrication techniques ...............................10

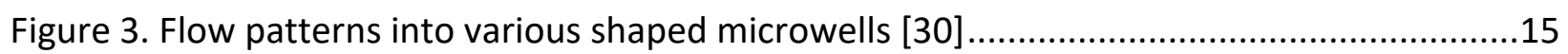

Figure 4. Oxygen inhibition layer prevents polymerization along the top and bottom of the PDMS

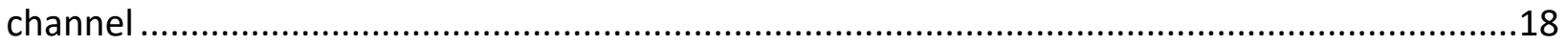

Figure 5. UV projection profile around microfluidic channel ............................................20

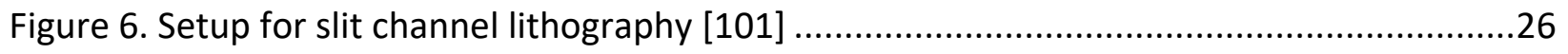

Figure 7. Microfluidics double channel: (a) Top channel. (b) Bottom channel with the microwell seating area. (c) Alignment of top and bottom channels....................................................31

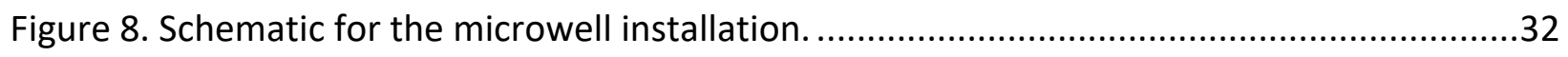

Figure 9. Photomask deigns: (a) Mesh and (b) Microwell. ..................................................36

Figure 10. Microwell fabrication using a $60 \mu \mathrm{m}$ tall channel...............................................3

Figure 11. Side view of: (a) Mesh layer (b) Microwell layer (c) Well with depth of $30 \mu \mathrm{m}$. (d) Top

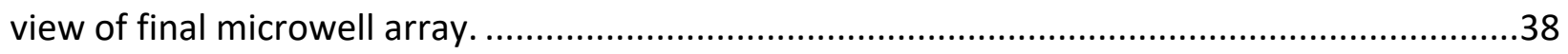

Figure 12. Fabrication schematic of sieved microwells with double layers using slit channel

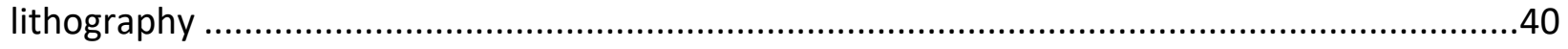

Figure 13. Microwells with an overall length of $3 \mathrm{~mm}, 100 \mu \mathrm{m}$ well diameter and $50 \mu \mathrm{m}$ pore length.

Figure 14. Microwells with different well diameters and shapes.....

Figure 15. Microwells with $100 \mu \mathrm{m}$ wells and mesh sizes controlled using different feature-sized photomasks

Figure 16. Mesh pore control with UV dose .45 
Figure 17. Assembled cross-flow microfluidic channel.

Figure 18. Installation of microwell using $60 \mu \mathrm{m}$ tall top channel

Figure 19. Installing a microwell through the outlet of a $60 \mu \mathrm{m}$-tall top channel......................50

Figure 20. Captured particles in $100 \mu \mathrm{m}$ diameter wells using a flow rate of $50 \mu \mathrm{L} / \mathrm{min} . \ldots \ldots \ldots . . .51$

Figure 21. Installation and capture of particles in $1300 \mu \mathrm{m}$ double channel ............................52

Figure 22. Fluorescent images of the particles captured using a flow rate of $50 \mu \mathrm{L} / \mathrm{min} . . \ldots \ldots \ldots . . . .53$

Figure 23. Fluorescent images of time lapse of particles captured using a flow rate of $\sim 9 \mathrm{~mL} / \mathrm{min}$ .54 


\section{List of Abbreviations}

$\begin{array}{ll}\text { SCL } & \text { slit channel lithography } \\ \text { SFL } & \text { stop flow lithography } \\ \text { PDMS } & \text { polydimethylsiloxane } \\ \text { PEG-DA } & \text { polyethylene glycol diacrylate } \\ \text { TMPeTA } & \text { trimethylolpropane ethoxylate triacrylate } \\ \text { DI } & \text { deionized }\end{array}$




\section{Introduction}

Microwells have become a popular tool for the isolation of single cells or particles. They are widely used for a range of biological and nonbiological applications because of their simplicity, high sensitivity and ease of analysis [1], [2]. In particular, they are commonly used to study the heterogeneity in a cell population. Traditional cellular studies measure the collective average of a population thereby limiting our understanding of how individual cells respond to their environment. Investigating these individual responses may provide insight into unseen biochemical functions and cellular mechanisms, which may be applicable to drug discovery, diagnostics, cancer research and regenerative medicine, among others [3], [4].

Numerous methods exist for the separation of cells from a large population [5], [6]. Some utilize a force gradient to either attract or repel cells for sorting such as dielectrophoresis (DEP) [7], optical tweezers [8] and magnetic tweezers [9]. In particular, flow cytometry may be used to separate select cells from a large population by measuring the differences in fluorescent dye emission as cells pass through an excitation laser [10]. All these methods are effective in separating singles cells from a larger population but require high energy input and exert an external force on the cells, which can have negative effects on cellular function. Additionally, microwells have numerous advantages, including: small sample size allowing for more assays to be run, parallelization resulting in faster overall analysis, lower material cost [11], faster response, high sensitivity due to small volumes [12], capability for longer term study due to cell immobility [13], and tandem microscopic analysis in the same focal plane [14]. These benefits 
make microwells popular for a wide range of applications, including single cell analysis [14]-[19], cell aggregation [20]-[26] and anti-counterfeiting applications [27], [28].

To seed the microwells a pipette is used to disperse a solution of cells or particles over the surface of the wells and is left for a period of time for them to settle into the wells [29]. This step is repeated several times to maximize occupancy, resulting in a time-consuming process. Gravity is the main driving force for sedimentation using this method, therefore the size and shape of the wells becomes an important factor for efficient trapping [30]. The shape and diameter tend to mimic the targets being captured while the depth is important to minimize the settling time [29], [31]. Using this static environment to load microwells results in several limitations like an extended settling times, the inability to efficiently exchange culture medium, and the lack of a shared chamber with a communications medium [32]. In order promote faster trapping, porous microwells are used with negative pressure [28] although the fabrication of these membranes is lengthy and challenging.

Microfluidics have been integrated with microwells to expedite sample seeding and sedimentation, and improve capture efficiency because microfluidics offers high throughput and small sample size [33]-[36]. Adding a microfluidic channel over the microwells reduces the sedimentation time since the cells or particles are more evenly dispersed over the surface of the wells, in addition to being nearer to the wells because of the low channel height [37], [38]. Also, moderate flow in microfluidic channels produce a low Reynolds number in the laminar regime, allowing for rapid media exchange within the devices, which is not possible in static environments [35]. 
Regardless, time is still required for the cells or particles to fully sediment into the wells since gravity is the main force driving them to the bottom of said wells [39]. To improve cell trapping, the flow patterns can be optimized to promote trapping, however, this can lead to complex studies [30], [40]. Alternatively, the introduction of porous microwells into microfluidic channels can take advantage of the properties of microfluidics to guide the targets into the wells using hydrodynamics.

A rapid fabrication method was developed, based on flow lithography techniques, which allows for easy customization of the mesh pore sizes in a two-step process. The microwells are inserted into $a$ in a microfluidic two-layered channel and single particles are caught using cross-flow trapping, The permeable wells act as a sieve, allowing fluid to pass through the open bottom of the wells while preventing the particles from following (Figure 1). This design eliminates the need for a sedimentation time since the hydrodynamic flow through the wells is the main driving force for particle capture. To fabricate these wells, slit channel lithography (SCL) is used, however this method has not been used to make differing heights within the same device, so a new process needs to be developed. Following fabrication, the microwells are installed in a microfluidic double channel to enable cross-flow trapping of particles to demonstrate the capabilities of the device. 
Top PDMS channel

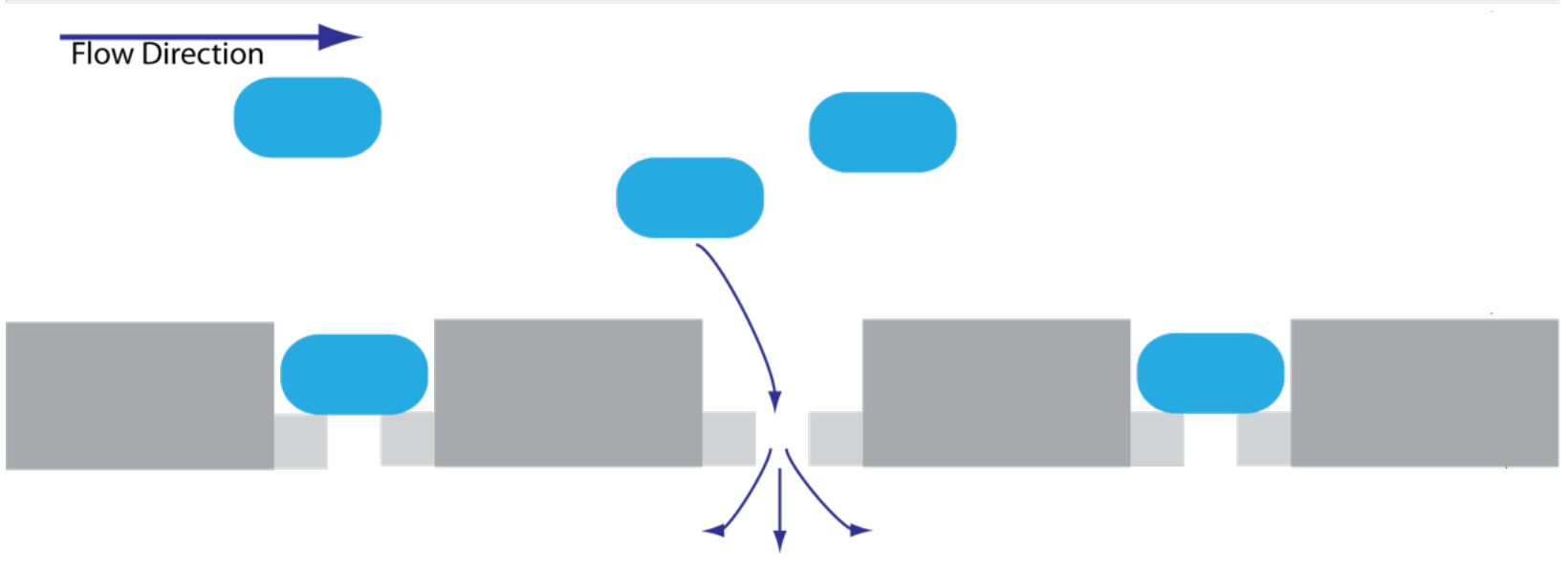

Bottom PDMS channel

Figure 1. Particles being captured in porous microfluidic device 


\section{Literature Review}

\subsection{Applications of Microwells}

Microwells have numerous applications for both biological and non-biological purposes by trapping both cells and particles. Not only do they have the capability to isolate and capture single cells from a large population, but they have frequently been combined with other biological tests to streamline processes. For instance, agarose-based microwells enables comet assays to be completed directly in those wells to examine DNA damage in a single cell [14]. Additionally, the confinement of cells makes microwells ideal for lysis because of its ability to keep all the cellular content within the wells for further analysis [34], [41] such as RNA sequencing and mutation analysis [16], [42], [43]. Microwells have been also adapted to study a cell's response to stimuli by studying their antigen production or oxygen consumption [44]-[48]; and to study cellular interactions with extracellular matrix (ECM) proteins by lining the surface of microwells with those proteins [32], [49]. The use of microwells for tissue engineering and tumor modeling has become another large area of focus, being used for the formation of embryonic stem cell aggregates with a controlled size, shape and homogeneity [50]-[54]. Microwells with dual surface properties, hydrophilic well-bottoms with hydrophobic sidewalls, have been implemented to create femtoliter droplets for cell analysis[55]. Lastly, microwell-based platforms have been developed to trap particles in specified geometric locations, allowing them to be encoded for anti-counterfeiting applications [56], [57]. The wide range of applications of microwells make this platform pertinent for point-of-care diagnostics and healthcare, thus meriting further study [58]. 


\subsubsection{Specific Applications of Microfluidic-Integrated Microwells}

The addition of microfluidics to microwells not only improves trapping but also adds the benefit of a wider range of applications. For example, microchambers can be assembled from a loaded array of microwells by layering a semi-permeable membrane over the array or by sealing the wells with oil, providing isolated conditions for contained cells [59]-[61]. Additionally, the trapping of cells within microfluidic channels streamlines the process for cultivating cell populations. In this case, microwells for single cell trapping line the channel bottom. Once fully seeded, a channel can be flipped over to transfer cells into larger wells for colony growth [62]. Lastly, static microwells are limited to a single medium since all wells are exposed to the same environment. This can be overcome in microfluidic-integrated-microwells by aligning a row of microwells with a series of fluidic channels enabling each row to be exposed to a unique medium. The use of channels eliminates the need for tedious solution changes, while avoiding the potential for cross-contamination between neighboring cells during this process [35]. Moreover, this can be applied to loading cells, where a variety of cell types can be loaded into individual rows within the same array. By rotating the fluidic channels perpendicularly to each well row, each cell can simultaneously be exposed to different media for experiment parallelization [63].

\subsection{Design Considerations for Microwells}

Although microwell platforms are guided by the same basic design and function; there are several variations regarding the arrangement, fabrication and operation of their arrays for cell or particle capture. Firstly, the geometry of the well must be optimized depending on the microwell 
application. Additionally, the means of fabrication and the material selection must ensure that the desired design and integrity of the platform is met.

\subsubsection{Well Geometry: Dimension and Shape}

When determining the well geometry, the most significant factors to consider are the well dimensions and shape. By customizing these for individual applications, it ensures that the wells can trap the desired number of cells or particles in each well [64].

Numerous studies have been conducted to determine the optimal height and width for a microwell. When used for single cell or particle trapping the well width and height must be large enough to capture a single target, but not so large as to allow multiple cells or particles from being trapped. Additionally, the wells must be tall enough to protect the trapped cells or particles from shear stress, which can cause the targets to be washed out. To achieve optimal trapping, the suggested ideal width of the well is approximately $10 \mu \mathrm{m}$ greater than the targeted cell or particle diameter and have an equal height-to-diameter ratio [29], [31]. Previous studies have focused on capturing single cells or particles, but by applying the same considerations, the dimensions of the well can be optimized for the desired number of cells.

Microwells can be made into various geometries, with cross-sections of circles, squares, triangles, rectangles, or spindles, some of which can be seen in Figure 2 [65]. For static microwells the geometry of the well is flexible in design, since gravity is the driving force to seed the cells or particles. Therefore, circular wells are commonly used due to the spherical shape of cells [30], although the shape of the well can be manipulated to promote trapping. For example, 
honeycomb shaped well-arrays have been designed with sloped walls into the well to direct the cells into the trap and prevent washout, in addition the well spacing is minimized to prevent cells from settling on the surface between the wells [66], [67].

\subsubsection{Microwell Materials}

There are numerous types of materials, namely silicon, optical fibers, and polymers, that are used to produce microwells. Among them, polydimethylsiloxane (PDMS) is the most popular choice since it is mouldable, flexible, transparent, electrically insulating, and permeable to gases [15], [30], [65]. Additionally, the use of polyethylene glycol (PEG) hydrogels is common for microwell applications because it is biologically inert and mechanically tunable by varying its polymeric length [20]. Agarose and polyacrylamide have often been used since they allow electrophoresis to be done directly in each microwell [14], [68]. For long-term cells studies, such as the examination of cellular differentiation and cell-based bioreactors, polyacrylamide is better suited than other hydrogels. Polyacrylamide has been shown to remain inert and support cell patterning for 28 days, a significantly longer period than with other support materials, such as agarose [69].

\subsubsection{Fabrication of Microwells}

Similar to well geometry, the fabrication method used is dependent on the application of the microwells, in addition to the type of construction material that is chosen. Photolithography is the most frequently used method, which can be applied to numerous types of materials. 
Standard photolithography consists of pouring photoresist on a silicon wafer, and spin coating the wafer to achieve a desired height, which corresponds to the depth of the microwells. The photoresist is exposed to UV light through a photomask and then placed in a developer to remove any uncured photoresist [70], [71]. Either the silicon mold is used directly as the microwell platform, or soft lithography is used to make a PDMS cast from the mold, the result of which can be seen in Figure 2a [64], [72]. Photolithography has also been applied to make PEG-based microwells in a grid array. This was done by combining PEG with a photoinitiator and polymerizing it with UV light focused through a photomask [20], [73]. This lithographic approach provides a simple pathway to produce microwells with greater control over their shape and dimension but suffers from a low production rate. Stamping is an alternative route to create microwells from hydrogels, namely polyacrylamide, agarose or PEG. A PDMS stamp is made using soft lithography and pressed into a droplet of hydrogel to form an array of wells. The platform is cured using a vacuum or UV light depending on the material [74]-[76].

Other than lithography techniques, there are several other methods that have been applied to produce microwell platforms. Etching is commonly used to fabricate wells on the surfaces of fiber optic filaments or silicone substrates. Chemical etching has also been utilized to produce microwells on the surface of optical fibers [77], [78], while deep reactive ion etching produces concave microwells on silicone [79], [80]. Additionally, injection molding has been employed to fabricate microwells made of polystyrene [81]. Another method uses thermal ablation to cut cylindrical holes into polyester with a $\mathrm{CO}_{2}$ laser. The polyester is mounted on a glass substrate using a silicone adhesive [82]. For larger microwell dimensions, where less resolution is required for the desired features, a 3D printed mold can be used. With stereolithography, the mold is 
printed to the desired specifications and used to stamp microwells into an agarose substrate. A significant advantage of this method is that it allows for more flexibility in the vertical direction to create tapered wells, which is difficult when using soft lithography [83].

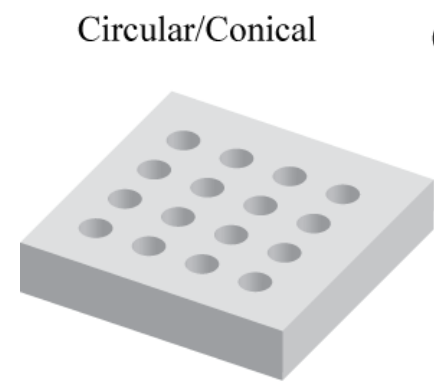

Square

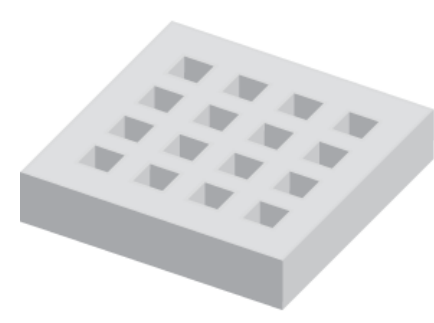

Triangular

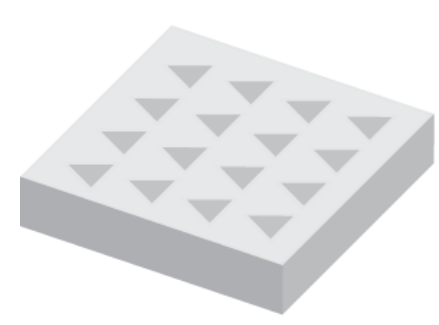

(a)

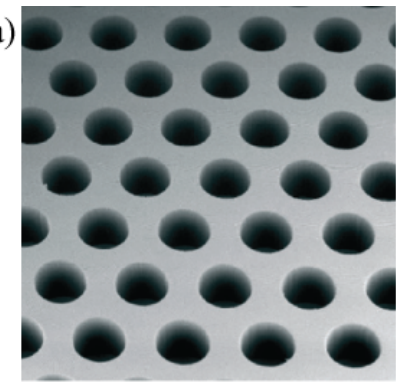

(c)

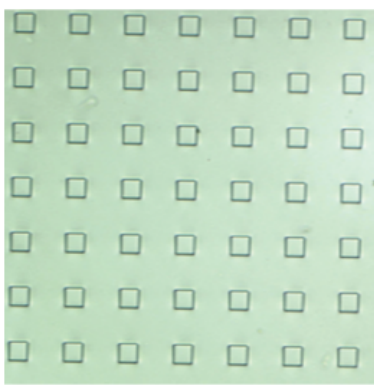

(e)

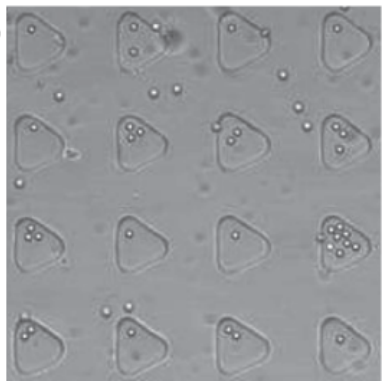

(b)

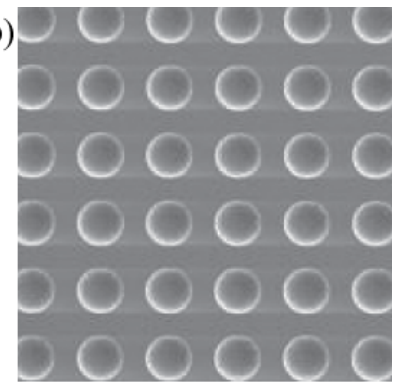

(d)

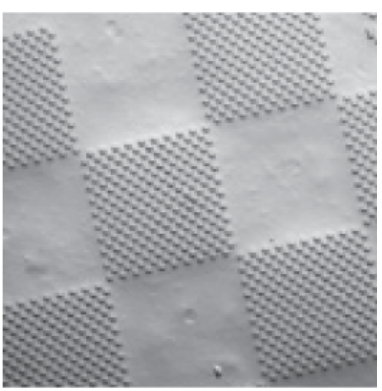

(f) 0000000 - 00.0000 - $000 \otimes 0$. - 000000 ๑ ब ब० 00 त० 0 아 0 $\circ 00000$

Figure 2. Examples microwells geometries and fabrication techniques. (a) SEM image of cylindrical microwells fabricated using soft lithography[62]. (b) SEM image of conical microwells fabricated using reactive ion etching [79]. (c) and d) Photomicrography of cubic microwells fabricated using soft lithography [84], [85]. (e) and (f) Photomicrography of triangular-prism microwells fabricated using soft lithography [30], [61].

An alternative pathway to create wells with curved bottoms, is the use of beads or particles as a template, which has been applied to create an array of concave shaped microwells in silicone. For this approach a layer of PDMS is poured over a single layer of particles and cured into an array 
of concave microwells (Figure 2b) [86], [87]. Lastly, CNC milling has been used to create deep conical wells into a polyoxymethylene block which is then used to create a PDMS mold used for stamping agarose into microwells. This milled stamps-based fabrication enables tapered shapes, which can be used for spheroid cell formation [88].

\subsection{Trapping Cells/Particles into Microwells}

Trapping cells or particles can happen in both static and fluidic environments, therefore a variety of techniques are used to achieve this. The effectiveness of the method and device are measured using the following parameters:

1. Capture or trapping efficiency- the ratio of the number of cells/particles introduced to the number of cells/particles trapped in wells [89];

2. Single cell occupancy- the number of wells filled with only a single target [29];

3. Filling rate- the number of wells filled with cells/particles compared to the total number of wells [90].

\subsubsection{Trapping Targets in Static Microwells}

Seeding in a static microwell is a simple process, although several techniques have been developed to improve it (Table 1). The main technique is based on sedimentation, but it has been enhanced in combination with a wiping method to force targets into individual wells. Other approaches that manipulate the surface properties and elasticity of PDMS for selective seeding are being developed. 
Table 1. Summary of Static Particle Capture Methods

\begin{tabular}{lcclc}
\hline $\begin{array}{c}\text { Trapping } \\
\text { Mechanism }\end{array}$ & $\begin{array}{c}\text { Diameter } \\
(\boldsymbol{\mu m})\end{array}$ & $\begin{array}{c}\text { Capture } \\
\text { Efficiency }\end{array}$ & \multicolumn{1}{c}{ Advantages } & Ref. \\
\hline Sedimentation & 25 & $\sim 85 \%^{*}$ & $\begin{array}{l}\text { Simple technique } \\
\text { No external forces }\end{array}$ & [29] \\
Wiping & 20 & & All cells deposited in wells & [89] \\
& 442 & $56 \%$ & & \\
Negative pressure & 229 & $74 \%$ & & Fast filling ( 60 s) \\
& $20^{+}$ & $\sim 76 \%^{* *}$ & Minimal cells lost during & \\
Well stretching & $15^{+}$ & $\sim 49 \%^{* *}$ & washing & Minimal cells lost during \\
& & $\sim 65 \%$ & washing
\end{tabular}

+Particle diameter $(\mu \mathrm{m})$; *Single cell occupancy; **Filling rate

For cell sedimentation, a suspension is added to the platform and then incubated from five minutes up to two hours for targets to sediment. To maximize well occupancy this process is repeated a second or third time [73], [92]. The length of time needed for cells to deposit into wells varies based on cell and well size as gravity is the only deposition force, thus deeper wells require longer settling times. Optimizing this parameter is important to ensure that wells are maximally filled [16]. To remove the excess medium and undocked cells, discontinuous dewetting is used: Excess solution is removed by tilting the microwell device to allow drainage by gravity or by removing it from a bath. This process results in an equal amount of solution remaining in each well due to differences in interfacial free energy between the substrate and the liquid [93], [94]. A second simple method uses wiping: A drop of suspension is pipetted along the edge of a cover glass, which is then slowly wiped along the surface of the microwell. The main 
advantage of wiping is that targets are directly deposited in the wells, and do not remain on the top surface of the platform [89].

Manipulation of the surface properties of PDMS has been used to selectively attach or repel cells. A UV sterilized PDMS surface has been found to be unsuitable for cell attachment, as demonstrated with human embryonic kidney cells, but they are able attach to a $\mathrm{O}_{2}$ plasmatreated PDMS surface with the only morphological change being elongation [84].

Additional methods to trap cells or particles exploits the elasticity of PDMS. One approach creates a stretchable microwell array that is capable of confining cells to wells. The fabricated microwell is stretched horizontally, from a diameter of 12 to $20 \mu \mathrm{m}$, by a plastic tube in which the cells flow through allowing them to settle into the expanded wells. After removing the tube, the microwells contract and trap the cells within them, preventing them from moving during analysis. After washing the loaded microwell, approximately $99.5 \pm 0.3 \%$ of cells remain in their wells, while only $70 \pm 20 \%$ of cells remained in standard microwells [91]. Similarly, using the flexibility of PDMS and creating a porous membrane layer at the bottom of the microwell, one method creates a driving force into the wells to trap particles and cells. Microwells designed to be slightly smaller than the particle dimensions can use vacuum suction through the pores at the well bottoms, coaxing polymeric particles to squeeze into said wells, locking them in place. By using larger-sized particles than the well diameter, the particles are locked into place, reaching a filling rate of $\sim 94 \%$ compared to $\sim 83 \%$ achieved when the particles are smaller than the wells. This $\sim 10 \%$ difference is due to particles lost during washing. When applied to cell capture, the time required to fill an 
array of 3800 was 100 seconds, which was a significantly reduction relative to sedimentation. This novel method has applications for sorting based on size, shape or modulus [28].

The utilization of static microwell arrays is straightforward, requiring no external instrumentation and minimizing external forces on the cell since gravity sediments them into the wells. The drawback of this method is that an extended period is required to fill an array given that this static approach will typically require several rounds of cell seeding and a period of settling for entrapment in individual wells.

\subsubsection{Trapping Targets in Microfluidic Integrated Microwell Systems}

Adding microwells into microfluidic channels involves new trapping mechanisms which have been summarized in Table 2 . The main advantage of integrating microwells into microfluidic channels is that they expedite particle or cell trapping into wells because of hydrodynamic forces in the channel. Numerous studies have been conducted to optimize trapping in this set up, mainly to understand the flow patterns within the channel. The streamline over the wells guides cells or particles towards the opening and as it approaches it loses velocity. The loss in velocity leads to gravity pulling it downward into the recirculation area of the well which traps the cell or particle in place [30]. By studying these flow patterns, shown in Figure 3, the triangular shaped wells have better recirculation in the wells. This geometry has been applied to capturing cells or particles with a $70-80 \%$ trapping rate [61]. Additional parameters have been modelled to optimize cell or particle capturing, including: the inlet velocity, particle diameter, particle density and channel design. Using triangular shaped pores, it was determined that a velocity of $0.01 \mathrm{~m} / \mathrm{s}$ in a long 
serpentine channel produced the highest capture rate. Also, trapping was increased when a larger number of particles were in the channel and the particles were larger in diameter [40].

Table 2. Summary of Trapping in Microfluidic Devices

\begin{tabular}{|c|c|c|c|}
\hline Trapping Mechanism & $\begin{array}{l}\text { Trapping } \\
\text { Efficiency }\end{array}$ & Advantages & Ref. \\
\hline Sedimentation & $70-90 \% *$ & No external forces & [37], [39] \\
\hline Sweeping & $>90 \%$ & $\begin{array}{l}\text { Faster filling ( } 5-10 \text { minutes) } \\
\text { Minimizes flooding }\end{array}$ & [38] \\
\hline Triangular wells & $70-80 \%$ & Possibility for colony formation & {$[30],[61]$} \\
\hline $\begin{array}{l}\text { Dielectrophoretic } \\
\text { forces }\end{array}$ & $60 \%$ & $\begin{array}{l}\text { Capable of capturing small } \\
\text { cells/particles }\end{array}$ & {$[95],[96]$} \\
\hline Magnetic forces & $62 \%$ & $\begin{array}{l}\text { High purity of trapped } \\
\text { particles/cells }(99.6 \%)\end{array}$ & [12] \\
\hline
\end{tabular}

*Filling rates
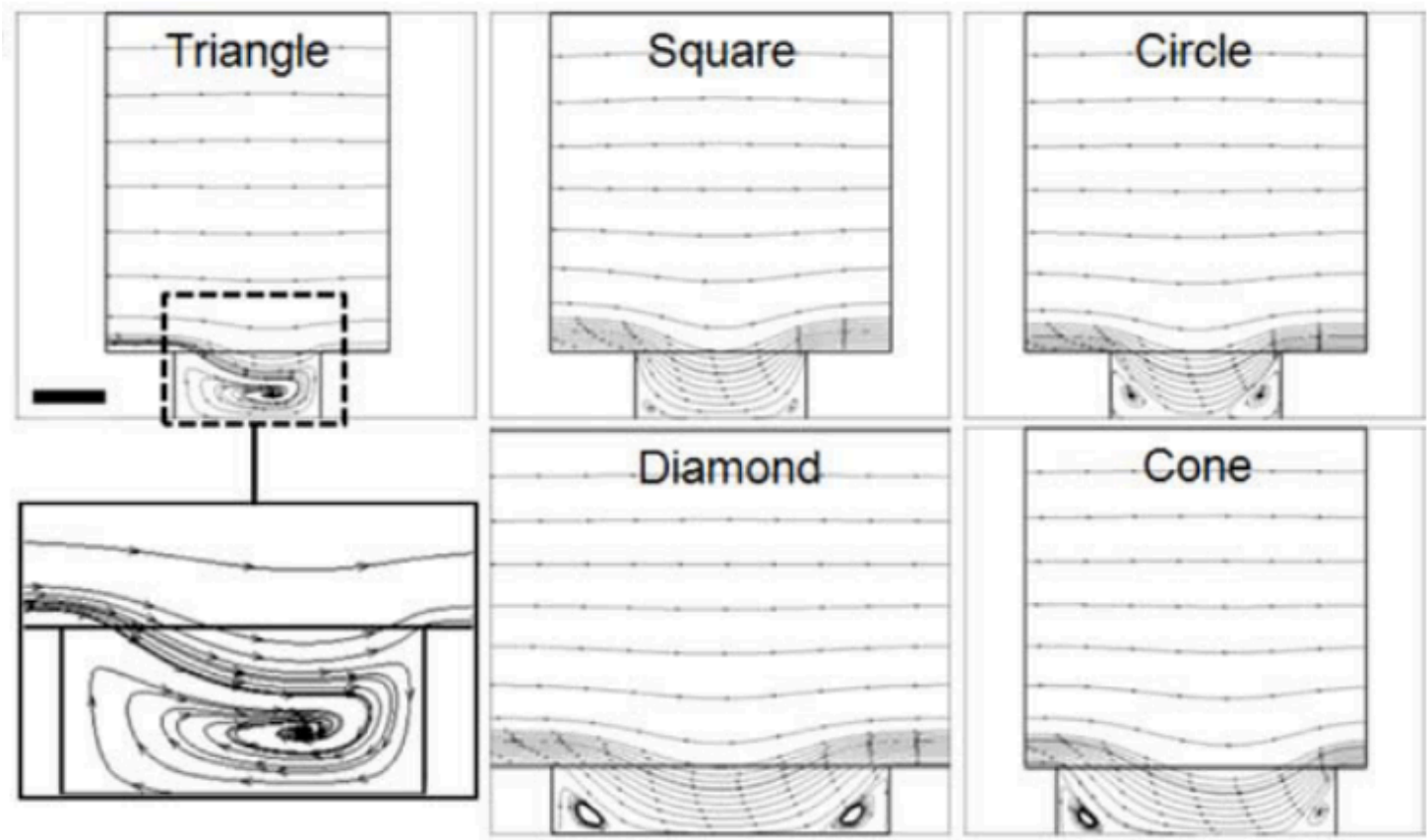

Figure 3. Flow patterns into various shaped microwells [30] 
While some studies have focused on the design of the wells, other studies have used standard circular microwells with adaptive trapping techniques to improve efficiencies. The most common method involves placing a microfluidic channel over an array of microwells and introducing the cells or particles into the channel through an inlet. The flow is paused in the channel to allow for the targets to settle into the wells. The sedimentation time is less than static wells because of the low channel height which brings the cells or particle into closer contact with the wells [37]. This process is repeated to maximize the number of wells that are filled. Lastly, a solution is flowed through the channel to remove any undocked cells or particles. This process typically results in 70-90\% of wells being filled [39]. A sweeping process is added to increase cell docking efficiency to $>90 \%$. A channel is filled with $30-40 \%$ of its' volume and then a small number of cells or particles are added. The meniscus of the solution forms plugs on both ends of the channel and by applying pressure to one end of the channel, the solution moves towards the opposite end. By alternating pressure between the two openings, the motion traps the targets into the microwells by lateral capillary forces [38], [97].

Additionally, external forces have been applied to promote trapping. Electroactive microwells have been used to trap bacterial cells using a strong electric fields [95], [98],[96], while magnetized microwells have been used to trap magnetic particles or immunomagnetic cells [12]. Although magnetic forces are advantageous over electric fields, since they are less damaging to cells, both methods still can have negative effects on cells, in addition to being limited to use on cells or particles that are reactive to these forces. 
Although different parameters are used to measure the effectiveness of the devices, it can be seen that microwells in microfluidic devices are more efficient in capturing particles as opposed to stand alone microwells. The addition of flow through the channel increases the trapping efficiency and reduces the time needed for sedimentation, making this an ideal method for single cell/particle isolation.

\subsection{Photolithography}

Photolithography involves exposing a polymer solution to UV light through a photomask, causing the exposed area to be polymerized into a replica of the photomask. The reaction involves the photolysis of a photoinitiator, in combination with a polymer, which separates to produce free radicals when exposed to UV light. The free radicals attach to the double bonds on end of the polymer chains creating a number of radicalized chains. From here three different reactions can occur: (1) the attached radical connects to another chain resulting in chain propagation, (2) two radicalized chains can attach to each other leading to chain termination, or (3) in the presence of oxygen the reaction is inhibited and stops [99]. The resulting mechanical properties of the hydrogel is dependent on the polymer structure, degree of crosslinking and the volume of the polymer. Therefore, a higher crosslinking density results in smaller mesh size with a stronger mechanical properties and lower swelling [100].

Stop flow lithography (SFL) combines photolithography in microfluidic channel to synthesize polymeric material into desired shapes, typically particles, in a continuous process [101]. A variation of this is called slit channel lithography $(\mathrm{SCL})$, where large sheets of polymeric material are polymerized into membranes. Hydrogels are commonly used for these purposes due to their 
biocompatibility. They are a polymeric material that forms a network of chains that become insoluble once crosslinked and are capable of absorbing large quantities of water [100].

The two techniques have similar set ups but vary in applications. Both use a PDMS microfluidic channel filled with prepolymer solution. When the flow is stopped an area of the channel is exposed to a light source, commonly UV light, through a specifically designed photomask. The area that is exposed to the light is polymerized, forming the structure. Due to the permeability of PDMS, a layer of oxygen is present at the top and the bottom of the channels which prevents polymerization (Figure 4). Therefore, when flow is resumed the layers act as a lubrication layer, allowing the polymerized material to easily down the channel [102].

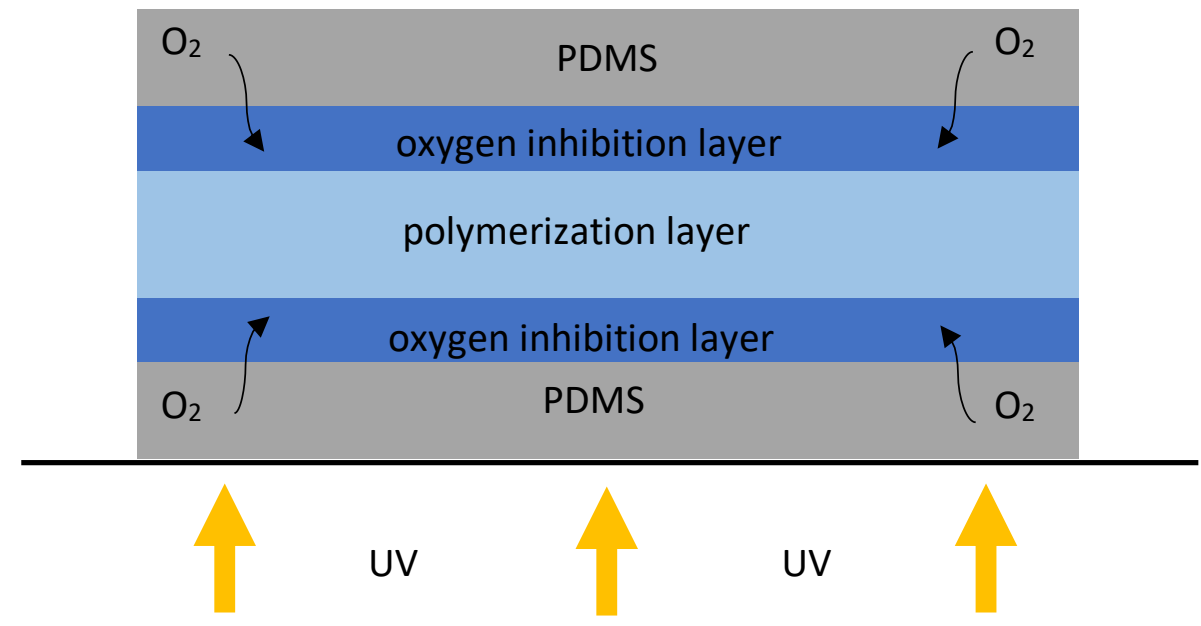

Figure 4. Oxygen inhibition layer prevents polymerization along the top and bottom of the PDMS channel

There are numerous parameters that go into controlling the shape and dimensions of the polymerized material. In the vertical direction the channel height is the primary control for the thickness of the polymerization. This is because the polymerization reaction occurs from the center of the channel and moves outwards until it stops at the oxygen layer at the top and bottom 
of the channel. Meanwhile, the main factor that goes into the determining the design of the polymerized material is the photomask. The area that is polymerized is the area that is exposed to the light, therefore areas that are blacked out create the pore features in the sheets. Additionally, the overall length of the sheet is defined based on the width of the photomask, although the objective can be used to change the resulting size from the photomask [102]. Higher objectives concentrate the light into a smaller area resulting in a smaller projection area, meanwhile lower objectives expand the area allowing for larger polymerizations. However, if constant power is supplied from the light source, the same light is being dispersed over a larger area requiring the UV light to be exposed for a longer period of time to achieve the same results. Additionally, the exposure time is important in maintaining the designed features and edges. If the light exposure time is not long enough, the polymer may have a low crosslinking density which can cause the structure to easily fall apart. Meanwhile, if the opposite happens and there is too much light exposure, the free radicals will continue to react with areas of the polymer material that is not exposed to light because of diffusion, resulting in over polymerization in both the vertical and lateral direction. Therefore, a median light exposure time is necessary to attain ideal features [103]. Finally, the focal plane is important for achieving straight edges in SCL. The UV profile is show in Figure 5 where the center of the projection is straight, and the tops and bottoms fan out with decreasing intensity. Therefore, it is important to find the optimal focus in the center of the projection area to achieve straight features. However, this straight portion varies based on the objective with the depth of field decreasing with decreasing objectives [104]. 


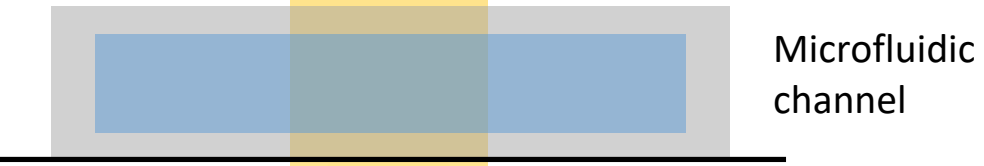

UV

Figure 5. UV projection profile around microfluidic channel

\subsection{Further Areas of Research}

Single cell capture is a transformative technique made possible with microwell arrays, allowing us to further elucidate cell population heterogeneity, and the properties of single cells within population ensembles. These arrays lead to numerous methods that can be used to analyze cells.

Traditional microwells are the most common technique currently used for single cell separation and will continue to be due to their simplicity. These platforms require limited equipment and a short learning curve, easing their use for a wide range of applications. However, devices with such simplicity have the caveats of longer settling times and lower trapping efficiency, as only gravity is drawing cells or particles into the wells. Ideally, to overcome this significant limitation, and without complicated designs, additional forces should be targeted to attract cells or particles into wells. By manipulating the surface properties of the microwells it is possible to attract certain 
cells or particles into the them. For example, a gradient surface designed with hydrophobic coatings between the wells with hydrophilic well walls [105].

Furthermore, the integration of microwells into microfluidic devices overcomes the restrictions of static microwells by using active trapping for cell or particle seeding. The application of hydrodynamic forces minimizes the required settling time but results in a more complicated platform. Although the introduction of microfluidics for cell or particle capture significantly improves the efficiency and customizability of trapping platforms, it also requires additional equipment and training compared to static microwells, thereby limiting its' mainstream usability. Removing the additional components, such as syringe or pressure pump to utilize microfluidicsintegrated microwells may accelerate its adaption to simple bench operation for biologists. Applying hydrostatic force to induce flow or using a portable pump with flow control could be one alternative solution.

Better control over cell types and their organization within an array would create a platform capable of multiple single cell analysis. Such a platform could sort a diverse cell population and selectively seed certain cells types of various quantities into microwells while simultaneously analyzing specific cells types after they have been seeded.

Although, the advancement of microwell-based technology has enabled controllable capturing and releasing of a group of or individual targeted cells for single cell analysis; however most efforts have been made for one specific size configuration. Both isolating and capturing cells from cell population with a wide range of size, shape, or combined could provide an additional tool to advance research fields dealing with such cells, for instance, fission yeast. Further, the 
ability to isolate and capture cells with specific n-th stages of the cell cycle from the first captured cells may accelerate mutation or aging research. 


\section{Materials and Methods}

There are several steps needed prior to capturing particles in microwells. To start microfluidic channels are fabricated using microfabrication techniques. These channels were used for the microwell fabrication and the double channels for cross-flow set up. Next, microwells are synthesized using SCL and particles are prepared using SFL which allows for rapid fabrication of materials in a continuous process. Finally, the double channels are assembled and the microwells are installed to assess their capture efficiency.

\subsection{Microfabrication of Microfluidic Moulds}

Photoresist (SU-8 2025) is poured onto a clean 4-inch silicon wafer and spin coated to create a thin, even layer. The acceleration, speed and length of spinning are selected for the desired feature height. Following the spin coating of the wafer, it is transferred to a hot plate and soft baked at $65^{\circ} \mathrm{C}$ and the cooled to room temperature. Next, the wafer is placed on a mask aligner and a photomask is centered over it. The photomask was previously designed on AutoCAD and printed on either a glass or film substrate. The wafer is exposed to UV light through the photomask. The length of exposure varies based on the output energy from the aligner and the desired thickness of the substrate. Following the exposure, the wafer is allowed to rest for ten minutes. Again, the wafer is transferred to a hot plate where it is hard baked at $65^{\circ} \mathrm{C}$ and then $95^{\circ} \mathrm{C}$ to fully set the SU-8 after exposure. Once the wafer is cooled, any unexposed photoresist is removed using a developer solution to expose the positive mold. For channels with two different heights, like the bottom channel of the double channel and the step channel for microwell fabrication, this process was repeated to create the second taller layer with special 
attention to proper alignment of the second photomask. The featured wafers are now used as master moulds to make PDMS channels with.

\subsection{Slit Channel Lithography Setup}

First, the microfluidic channels are made from the master mould. A 10:1 ratio of poly(dimethylsiloxane) (PDMS) to curing agent was weighed out and mixed for $1 \mathrm{~min}$ to thoroughly combine the two. The mixture was placed in a partial vacuum for $1 \mathrm{~h}$ until all the bubbles are removed. The PDMS is poured onto a cleaned master mold close to the wafer's surface to avoid bubbles from forming. Once the mold is filled, any bubbles are removed using a transfer pipette to avoid air pockets in the cured channels. The mold is then baked at $65^{\circ} \mathrm{C}$ for at least 1 hour. After coming back to room temperature, the PDMS is cut out from the mold using a scalpel along the edges and separated into individual channels. Any dust is removed using tape and inlets are cut using a $1 \mathrm{~mm}$ biopsy punch and $1 \mathrm{~cm}$ wide outlets are cut using a scalpel. The channels are attached to glass slides using partially cured PDMS. To coat the glass slides with PDMS, a small amount of PDMS is sandwiched between two glass slides and the two slides are pulled in opposite directions to evenly coated the surface of the bottom slide. A smooth surface is important to ensure that a constant height is maintained throughout the channel. The slide is partially cured at $65{ }^{\circ} \mathrm{C}$ for 15 mins to attain a tacky surface for the channel to adhere to. The prepared channels are placed on the PDMS slides and pressed along the edges to seal well. The slides are placed back in the oven at $65^{\circ} \mathrm{C}$ for at least $1 \mathrm{~h}$ to bond the channels fully to the slide.

A sealed pipette tip is made to dispense the prepolymer solution into the channel. A $200 \mu l$ pipette tip is placed in a small petri dish filled with $1 \mathrm{~cm}$ of PDMS, with the tip facing upwards, 
and is cured at $65^{\circ} \mathrm{C}$ for $1 \mathrm{~h}$. Once fully cured, the pipette tips are carefully removed from the PDMS to keep the PDMS cap on the pipette tip. A $1 \mathrm{~mm}$ hole is punch using a biopsy punch through the PDMS. Using a $1 \mathrm{ml}$ syringe, both the channel and pipette tip is filled with prepolymer solution and the pipette tip is inserted into the inlet of the channel.

Using the hole at the top of the pipette tip, $1 \mathrm{~mm}$ tubing fitted with a 19-gauge needle tip is connected to the channel setup. The tubing is connected to a three-way solenoid valve used to start and stop flow in the channel using pressurized air. The valve was connected to an air source through a pressure regulator. The channel is placed on an inverted microscope and the light source is projected from underneath the channel which is controlled using a shutter. When the shutter is open the light passes through a photomask and objective before being exposed to the channel. LabView was previously set up in the lab to control the shutter and valve. The exposure time, which is the time between opening and closing the shutter, can be set in LabView, in addition, to setting the amount of time the valve is opened, which exposes the channel to pressurized air, and the amount of time the valve is closed, which exposes the channel to atmospheric air. Figure 6 shows the arrangement of the equipment.

To determine the focal plane in the channel, the edge of the channel is located and brought into focus. A series of practice polymerizations are made along the edge and each time the focus is changed incrementally using the fine focus knob to move up and down from the focal plane. The focus that produces the sharpest features, i.e. corners and pore shapes, is used as the focus for the experiment. This process is done prior to starting $\mathrm{SCL}$ and changes based on the objective and channel height. 


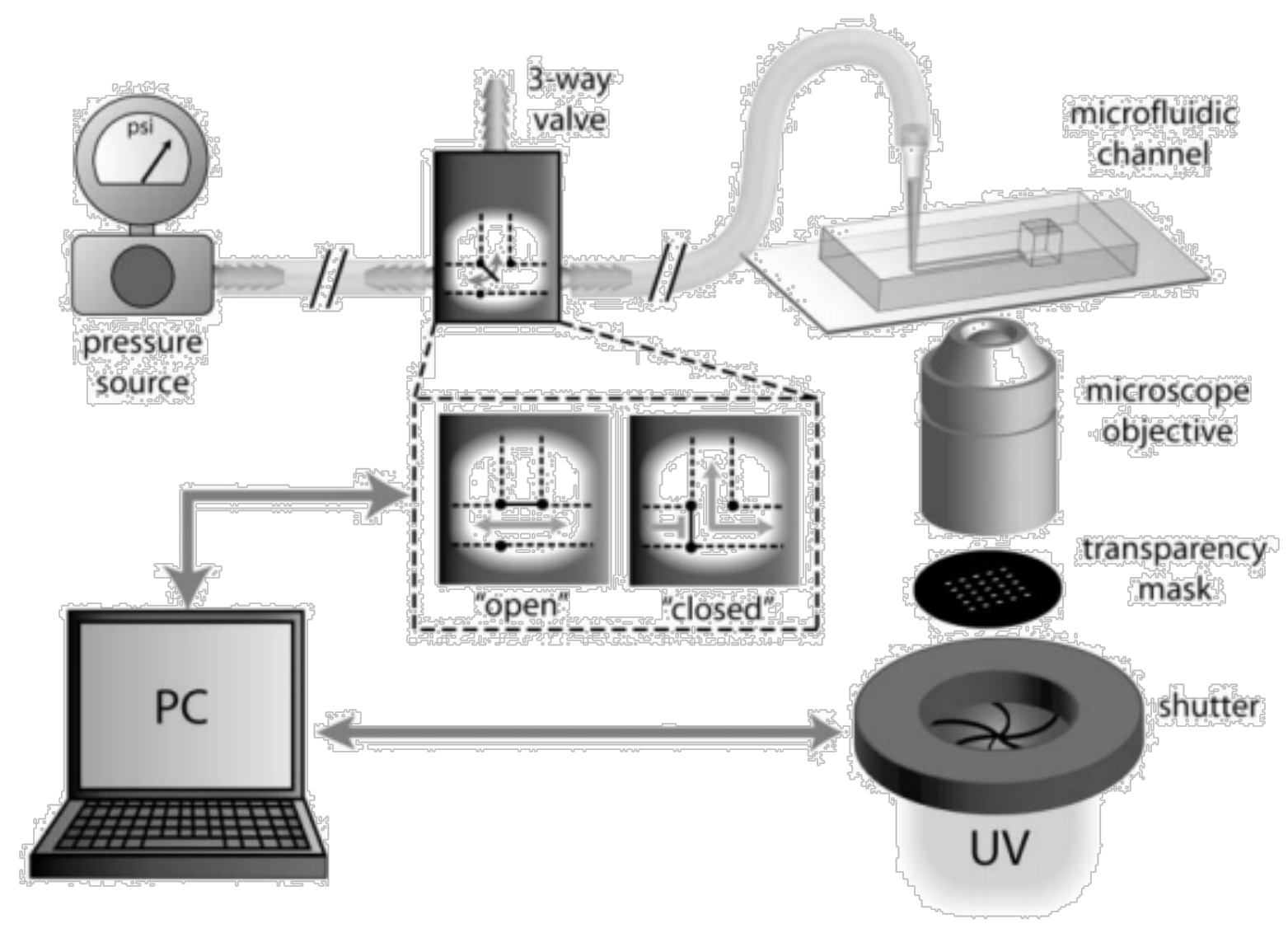

Figure 6. Setup for slit channel lithography [101]

\subsubsection{Sieved Microwell Fabrication}

The prepolymer solution to fabricate the microwells was $47.5 \%$ poly(ethylene glycol) diacrylate (PEG-DA 700), 47.5\% trimethylolpropane ethoxylate triacrylate (TMPeTA 428) and 5\% 2-hydroxy2-methylpropiophenine photoinitiator (Darocur 1173) by volume. The materials were weighed in a Falcon tube using an analytical scale because of difficulty accurately pipetting the viscous hydrogels. After combining the solution, it was vortexed for at least 1 min to properly combine the TMPeTA and PEG-DA and sonicated to remove any bubbles that formed from mixing. If using 
a pre-prepared solution, it was vortexed and sonicated prior to use to ensure uniform composition since the TMPeTA and PEG-DA have a low miscibility and separated over time.

The photomasks for the mesh and microwell layers were designed in AutoCAD and printer on transparencies. The UV light source is connected to the microscope and set to $80 \%$ output. The UV light has a wavelength of $365 \mathrm{~nm}$, which corresponds to the excitation energy of the selected photoinitiator. A channel with two different heights is used, the first area has a height of $25 \mu \mathrm{m}$ and further down the channel the height increases to $60 \mu \mathrm{m}$. LabView is set to 'Pulse v2' mode, with an exposure time of $200 \mathrm{~ms}$, valve on delay of $100 \mathrm{~ms}$ (valve closed), and valve off delay of $300 \mathrm{~ms}$ (valve open).

After finding the optimal focus in the $25 \mu \mathrm{m}$ tall area of the channel, actuate is pressed in LabView. The shutter opens exposing the area to UV light through the photomask and the $10 \times$ objective for $200 \mathrm{~ms}$. Following the exposure, there is a pause for $100 \mathrm{~ms}$ and then flow is resumed in the channel for $200 \mathrm{~ms}$ at a pressure of $10 \mathrm{psi}$ to move the mesh layer to the $60 \mu \mathrm{m}$ tall area of the channel. Next, the photomask is switched to the microwell photomask with the triangle in the photomask pointing to the right. The focal area is centered over the mesh layer and using the same parameters as previously, the microwell layer is polymerized over top of the mesh. When the flow is resumed in the channel, the microwell is pushed into the outlet.

The microwells are transferred from the outlet of the channel to an Eppendorf tube using denatured alcohol (Histoprep 95\%). The microwells are vortexed for 15 secs and the alcohol is removed from the Eppendorf using a pipette and fresh alcohol is added with a new pipette tip. This is done three times to wash excess prepolymer solution from the microwells. The same 
process is repeated using a solution of deionized water with $5 \% \mathrm{v} / \mathrm{v}$ polyethylene glycol sorbitan monolaurate (Tween 20). The microwells are stored in this final aqueous solution for later use.

To make microwells using the $5 \times$ objective, a violet light source at $100 \%$ power with a wavelength of $405 \mathrm{~nm}$ is replaced as the light source. Additionally, the prepolymer solution is changed to 49.5\% PEG-DA 700, 49.5\% TMPeTA 428 and 1\% phenylbis(2,4,6-trimethylbenzoyl)phosphine oxide photoinitiator (Irgacure 819). To prepare the prepolymer solution a 1:1 mixture of PEG-DA 700 and TMPeTA 428 is prepared and the powder photoinitiator is mixed into it using a vortex. Excess photoinitiator settles to the bottom of the Falcon tube and only the top portion of the solution is used. The same process is used as the $10 \times$ objective, however different parameters are set in LabView which are shown in Table 3.

Table 3. Operating Conditions for Slit Channel Lithography

\begin{tabular}{|c|c|c|c|c|c|}
\hline & $\begin{array}{l}\text { Mesh } \\
\text { Layer }\end{array}$ & $\begin{array}{l}\text { Microwell } \\
\text { Layer }\end{array}$ & $\begin{array}{l}\text { Mesh } \\
\text { Layer }\end{array}$ & $\begin{array}{l}\text { Microwell } \\
\text { Layer }\end{array}$ & Particles \\
\hline Objective & \multicolumn{2}{|c|}{$10 x$} & \multicolumn{2}{|c|}{$5 \times$} & $20 x$ \\
\hline Light Source & \multicolumn{2}{|c|}{ UV (365 nm) } & \multicolumn{2}{|c|}{ Violet (405 nm) } & UV (365 nm) \\
\hline \multicolumn{6}{|l|}{ Composition (\%v/v) } \\
\hline PEG-DA 700 & \multicolumn{2}{|c|}{47.5} & \multicolumn{2}{|c|}{49.5} & 95 \\
\hline TMPeTA 428 & \multicolumn{2}{|c|}{47.5} & \multicolumn{2}{|c|}{49.5} & - \\
\hline Darocur 1173 & \multicolumn{2}{|c|}{5} & \multicolumn{2}{|c|}{ - } & 5 \\
\hline Irgacure 819 & \multicolumn{2}{|c|}{-} & \multicolumn{2}{|c|}{$\sim 1$} & - \\
\hline Overall Length $(\mu \mathrm{m})$ & 1000 & 1500 & 2000 & 3000 & $40-90$ \\
\hline Channel Height $(\mu \mathrm{m})$ & 25 & 60 & 25 & 60 & 30 \\
\hline \multicolumn{6}{|l|}{ LabView Settings } \\
\hline Mode & Pulse v2 & Pulse v2 & Pulse v2 & Pulse v2 & Continuous \\
\hline Exposure Time (ms) & 200 & 200 & 25 & 25 & 30 \\
\hline
\end{tabular}




\begin{tabular}{rccccc}
\hline & $\begin{array}{c}\text { Mesh } \\
\text { Layer }\end{array}$ & $\begin{array}{c}\text { Microwell } \\
\text { Layer }\end{array}$ & $\begin{array}{c}\text { Mesh } \\
\text { Layer }\end{array}$ & $\begin{array}{c}\text { Microwell } \\
\text { Layer }\end{array}$ & Particles \\
\hline Valve Open (ms) & 300 & 300 & 500 & 500 & 50 \\
Valve Closed (ms) & 100 & 100 & 100 & 100 & 100 \\
Delay (ms) & - & - & - & - & 500 \\
Air Pressure (psi) & 10 & 10 & 12 & 12 & 12 \\
\hline
\end{tabular}

\subsubsection{Particle Fabrication using Stop Flow Lithography}

Cylindrical particle are made using the same set up as previous, however they are produced continuously using SFL [101]. A prepolymer solution of 95\%v/v PEG-DA 700 and 5\%v/v Darocur 1173 is prepared in the same way as the microwell prepolymer solution. To make fluorescent particles $5 \%$ Rhodamine $B$ acrylate (1\% in DI water) is added to the particle prepolymer solution prior to polymerization. The channel used is $2 \mathrm{~mm}$ wide and with a height of $30 \mu \mathrm{m}$ with $1 \mathrm{~mm}$ inlets and outlets. The inlet is connected to the air source, while the outlet has $1 \mathrm{~mm}$ tubing connected to an Eppendorf tube to collect the particles. Different photomasks are used depending on the diameter of the desired particles, ranging from 40 to $90 \mu \mathrm{m}$. The optical focus is found in the channel using the $20 \times$ objective. The depth of field is narrower with this objective so smaller incremental changes are made using the focal knob in order to find the focus.

LabView is set to 'Continuous' mode, with an exposure time of $30 \mathrm{~ms}$, valve on delay of $200 \mathrm{~ms}$, valve off delay $50 \mathrm{~ms}$ and a delay of $500 \mathrm{~ms}$ (Table 3). After pressing actuate in LabView, the following occurs: (1) a delay of 500 ms to allow flow to stop, (2) particles are polymerized with UV light for $30 \mathrm{~ms}$, (3) there is a pause for $100 \mathrm{~ms}$, (4) the air valve is opened for $50 \mathrm{~ms}$ at a 
pressure of $12 \mathrm{psi}$ to move the particles out of the focal area. The process is repeated continuously for at least $1 \mathrm{~h}$ with the pipette dispenser being refilled with prepolymer solution throughout the process. The particles are collected in the Eppendorf tube, which is filled with denatured alcohol to prevent the particles from sticking together. Both the Eppendorf and the pipette leading to the channel are wrapped in aluminum foil to prevent unwanted polymerization during operation.

The particles are washed four times using denatured alcohol and 5\% Tween 20 in deionized (DI) water. The same washing process is used as the microwells; however, a centrifuge is used after the particles are vortexed to sediment them to the bottom of the Eppendorf tube faster.

\subsection{Assembly of Microfluidic Double Channels}

The double channel consists of two microfluidic channels that are attached perpendicularly, as seen in Figure 7. Two different methods are used to assemble the double channels depending on the height of the top channel.

\subsubsection{Top Channel with 60 um Height}

Using the same method as for the previous channels, a 10:1 ratio of PDMS is prepared and poured into the top and bottom channels master mold. Both channels are placed in the oven at $65{ }^{\circ} \mathrm{C}$ for 30 minutes to partially cured the channels. The channels are removed from the oven and allowed to cool before cutting out the channels with a scalpel. The channels are peeled off slowly from the mold to prevent stretching of the channels. Two $1 \mathrm{~mm}$ inlets and a $1 \mathrm{~cm}$ outlet is cut on the top channel using biopsy punches. Dust is removed from the top and bottom channel using tape 
and the channels are aligned using a stereo microscope. The top channel is placed perpendicularly to the bottom channel, with the edges of the top channel aligning perfecting with the edges of the seating area in the bottom channel. Once perfectly aligned, the edges of the two channels are pressed together to ensure proper sealing of the two channels. The channel is placed in the over at $65^{\circ} \mathrm{C}$ for at least $1 \mathrm{~h}$ to completely cure the two layers. Finally, the channel is cooled to room temperature before using. Using a biopsy punch, two $1 \mathrm{~cm}$ holes are cut through the top layer of PDMS as outlets for the bottom channel.

a

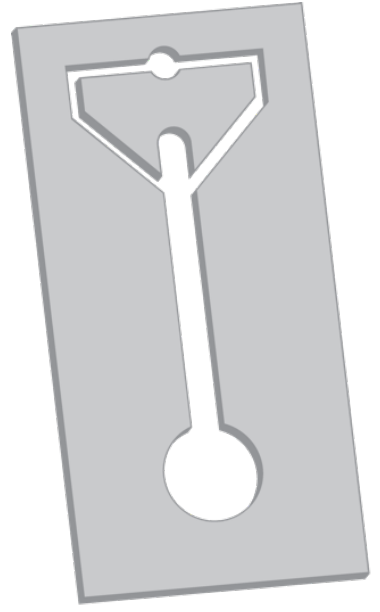

b

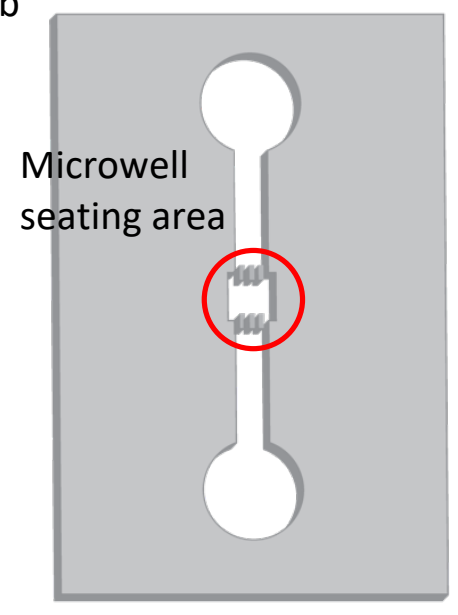

C

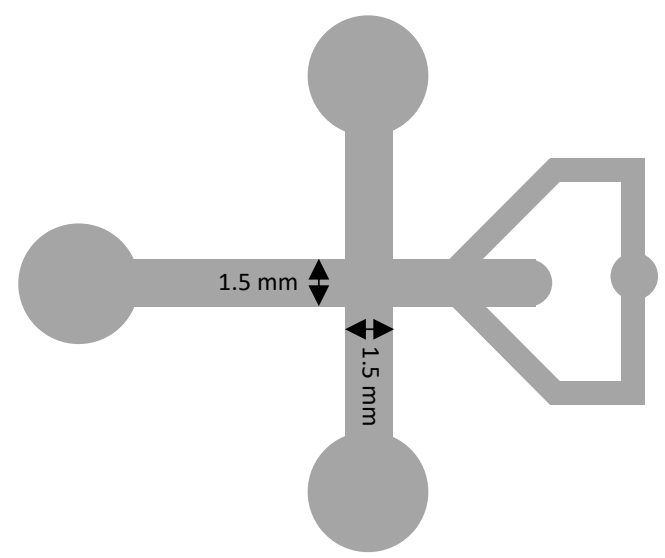

Figure 7. Microfluidics double channel: (a) Top channel. (b) Bottom channel with the microwell seating area. (c) Alignment of top and bottom channels.

\subsubsection{Top Channel with 30 um Height}

The top and bottom channel master molds are filled with a 10:1 ratio of PDMS and are fully cured at $65{ }^{\circ} \mathrm{C}$ overnight. The channels are cut from the mold and peeled off, the inlet and outlet holes are cut in the top channel using a biopsy punch and any dust is removed using tape. Using a 200 $\mu \mathrm{L}$ pipette tip with $1 \mathrm{~cm}$ of the tip cut off, a microwell was removed from the Eppendorf. The 
pipette was repeatedly pushed up and down to cause the microwells to float in the solution, and finally one microwell was removed and placed in the bottom channel near the seating site. Using the orientation of the triangular pores, the microwell is flipped so that the wells are facing upwards and placed in the center of the seating area. Using a new pipette tip, excess solution is removed from the surface of the channel. The channel is placed in a desiccator for 15 mins to shrink the microwell and remove any liquid. Again, using tape any dust is removed without moving the microwell. Both the top and bottom channels are placed in a plasma chamber at 20 Watts for $30 \mathrm{~s}$. Again, the channels are aligned using a stereo microscope, although the top channel width is $200 \mu \mathrm{m}$ than the bottom channel so alignment is not as important. The channel is placed in the oven at $65^{\circ} \mathrm{C}$ for at least $1 \mathrm{~h}$. Two $1 \mathrm{~cm}$ outlets are cut for outlets of the bottom through the top layer of PDMS (Figure 8).

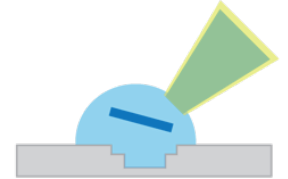

1. Pipetting the microwell onto the bottom channel

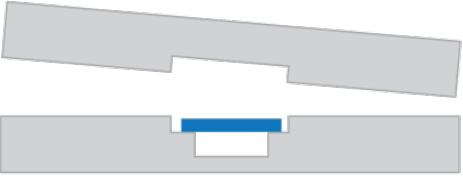

5. Bonding the channels together

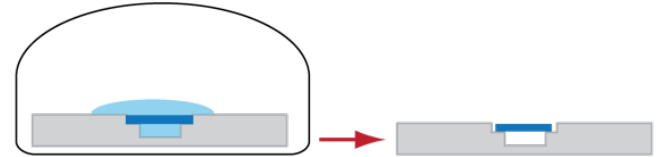

2. Vacuum-drying the microwell in a desiccator Dried and shrunked microwell once removed

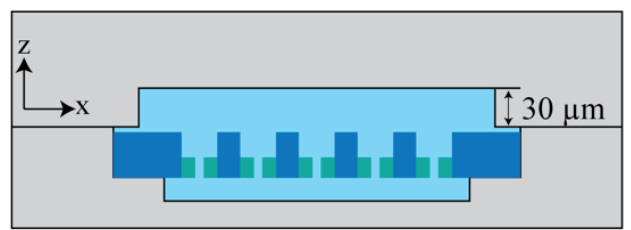

6. Filling the bonded chanel with the buffer solution and the swollen microwell

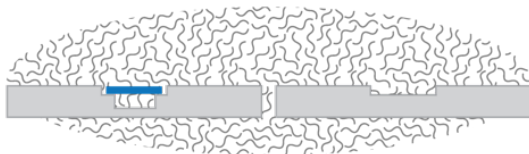

4. Plasma the bottom and top channels

Figure 8. Schematic for the microwell installation: the microwell array is loaded onto the open bottom channel and liquid is evaporated using partial vacuum. The top channel, with a $1300 \mu \mathrm{m}$ length and $\mathbf{3 0}$ $\mu \mathrm{m}$ height, and bottom channels are plasma treated ( $20 \mathrm{~W}$ for $30 \mathrm{~s}$ ) and aligned to sandwich the microwell in place.

\subsection{Particle Capture Setup}


A $3 \mathrm{ml}$ syringe is filled with 5\% Tween 20 and DI water and any air bubbles in the syringe are pushed out. The syringe is fitted with a 20 gauge need tip and the solution is pushed through the tip to ensure there was no air in the syringe. Using the top inlet, the double channel is filled with the solution and checked for bubbles. If any bubbles were present the solution was removed using air and the solution was reintroduced until the channel is bubble free. Depending on the channel, if there was no microwell installed, then the microwell was inserted through the top channel.

Using a cut pipette tip, the microwell was transferred from the Eppendorf to the outlet of the top channel. The same technique as previously was used to pick up a microwell from the solution. Once a microwell was inserted into the outlet, the microwell was flipped to ensure the wells were facing upwards. The microwell is pushed into the top channel and using the syringe, a small burst of solution is used to flow the microwell into the intersection. To ensure a proper installation in the cross-section, the back of a bent syringe tip is pushed against the top of the channel at the intersection to fit the microwell in the slot. The channel is placed on a glass slide on the stage of the microscope.

After installing the microwell into the microfluidic channel, two syringe pumps are attached to the inlets of the channel. A $3 \mathrm{ml}$ syringe is filled 5\% Tween 20 and DI water and a second is filled with $1.5 \mathrm{ml}$ of $5 \%$ Tween 20 and $\mathrm{DI}$ water and $1.5 \mathrm{ml}$ of particles. Prior to drawing up from the 2 $\mathrm{ml}$ Eppendorf the particles are stored in, they are vortexed to disperse the particles. Any bubbles are removed from the syringe by tapping the side of the syringe. Both syringes are fitted with 1 $\mathrm{mm}$ tubing and a 19-gauge needle tip at the end, and the solution is pushed through the tubing 
before inserting the syringes into the syringe pumps. The syringe pumps are started before inserting the tubing into the channel to prevent air bubbles from forming. The syringe containing the $5 \%$ Tween 20 solution is inserted into the top inlet and the particle syringe is inserted into the other inlet. Once inserted, the flow rates were adjusted for the experiment.

To reuse the particles and microwells after the experiment, the particles were collected into a Falcon tube and the microwell was pushed to the outlet of the top channel and stored in an Eppendorf. The double channels were stored in a closed container to prevent dust from getting in the channel.

\subsection{Image Processing}

Bright-field and fluorescence images of the microwells and PDMS devices were taken using an inverted microscope with $5 \times$ objective lenses, and a digital SLR camera. Fluorescent is added to differentiate between the particles and microwells. Without the fluorescent in the particles are transparent making it difficult to determine their exact placement. The images taken are then used to visually count the number of wells and the number of particles captured into the wells which is used to calculate the capture efficiency:

$$
\text { Capture Efficiency }=\frac{\text { No. of wells filled }}{\text { Total number of wells }}
$$




\section{Results and Discussion}

\subsection{Fabrication of Sieved Microwells}

Slit channel lithography was used to fabricate the sieved microwells. The wells were designed to be porous on the bottom of each well to allow flow through the bottom of the pores and draw in particles using hydrodynamic flow. In order to fabricate the wells using SCL, two separate layers are needed. The first layer would be the mesh at the bottom of the wells, stopping the particles from passing through the wells. The second layer is over top of the mesh layer and contains the actual wells for the particles. In order to have wells that are deep enough to keep the particles, the mesh layer needs to be shorter than the microwell layer. The depth is important to prevent the particles from washing out during operation. According to literature a $10 \mu \mathrm{m}$ difference between the particle height and well depth is optimal, however, in this arrangement a hydrodynamic flow with help keep the particles in place so this margin is not as important.

The two photomasks were designed prior to starting using AutoCAD (Figure 9). The microwell layer was designed to be $1500 \mu \mathrm{m}$ in length with an array of circular pores in the center. One of the pores was replaced with a triangle to establish the orientation of the wells when installing. The mesh layer was a grid of square pores with an overall circular shape to make alignment of the second layer easier. Prior to starting, a few of the polymerization parameters were set for the experiment, including the 10x objective, polymer composition, UV light source and $80 \%$ power output. The objective and composition were selected because these provided the desired length and material properties, meanwhile the power output was set by the manufacturer. Therefore, the remaining adjustable parameters were the channel height and exposure dose. 

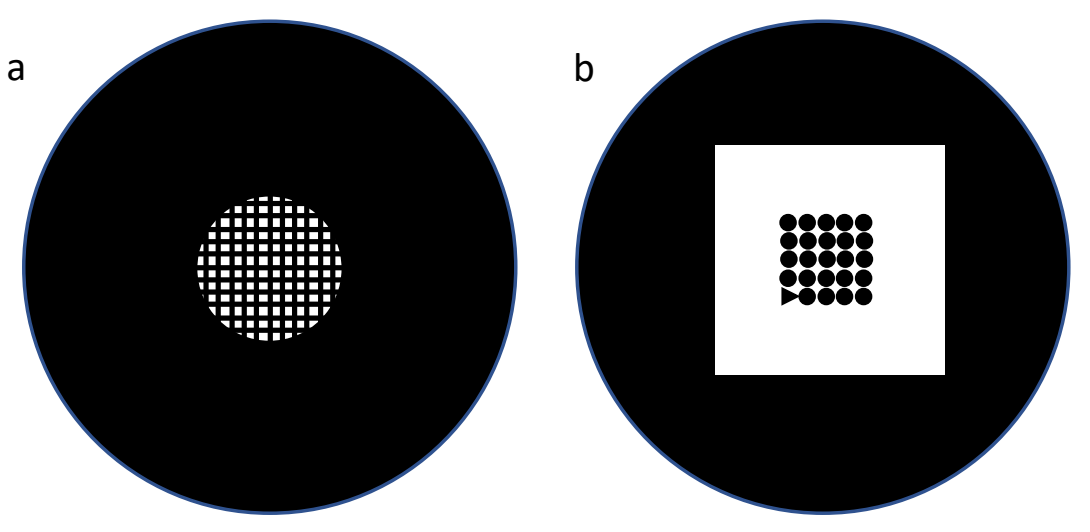

Figure 9. Photomask deigns: (a) Mesh and (b) Microwell.

The first attempt at making sieved microwells fabricated them using a straight channel with a constant height of $60 \mu \mathrm{m}$. To achieve the height difference between the mesh and microwell layer, it was assumed that decreasing the UV exposure time of the mesh layer would result in a thinner layer which would sink to the bottom of the channel after polymerization. This would create the change in height that is needed to create the well depth, as shown in Figure 10. Based on the conditions set, the optimal UV exposure time was 200 ms so it was assumed that an exposure time of 150 ms would produce a thinner mesh layer. However, actual application of the microwells resulted in no particles being captured. This could have been due to the mesh layer not being thin enough to make a significant height difference in the wells. Reducing the exposure time further was not possible because the already low cross-linking density was creating a fragile mesh layer which often breaking prior to installation.

a

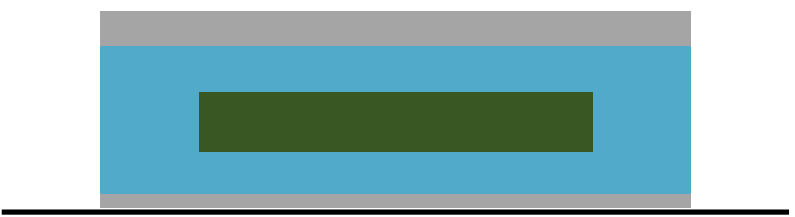




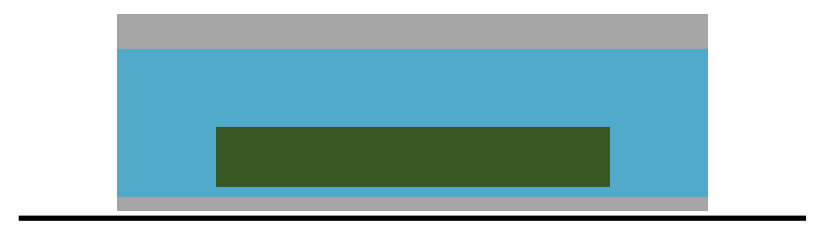

Mesh layer

C

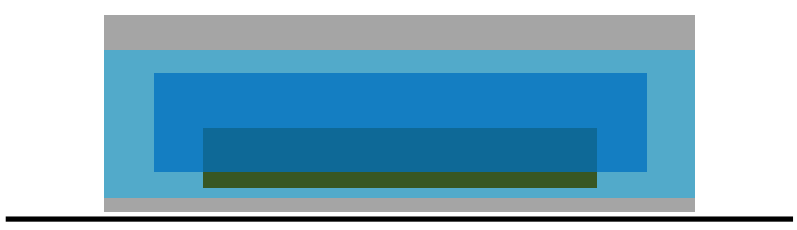

PDMS

Prepolymer solution

Microwell layer

Figure 10. Microwell fabrication using a $60 \mu \mathrm{m}$ tall channel. (a) Shorter mesh layer is polymerized first using a shorter exposure time. (b) The mesh layer sinks to bottom of channel. (c) Microwell layer is polymerized over top.

In order to gain better control over the height and rigidity of the mesh layer, the two layers were made in separate channels. The mesh layer was polymerized in $25 \mu \mathrm{m}$ tall channel first, then transferred to a $60 \mu \mathrm{m}$ tall channel to polymerize the microwell layer over top of it. Figure 11 shows the resulting heights in the channel. The mesh layer had a height of $\sim 20 \mu \mathrm{m}$, the microwell layer had a height of $\sim 55 \mu \mathrm{m}$ and the well depth was approximately $\sim 30 \mu \mathrm{m}$. As mentioned, the polymerization heights are controlled using the channel height. The expected height is approximately $5 \mu \mathrm{m}$ less than the channel height due to the oxygen inhabitation layer at the top and bottom of the PDMS channel. 

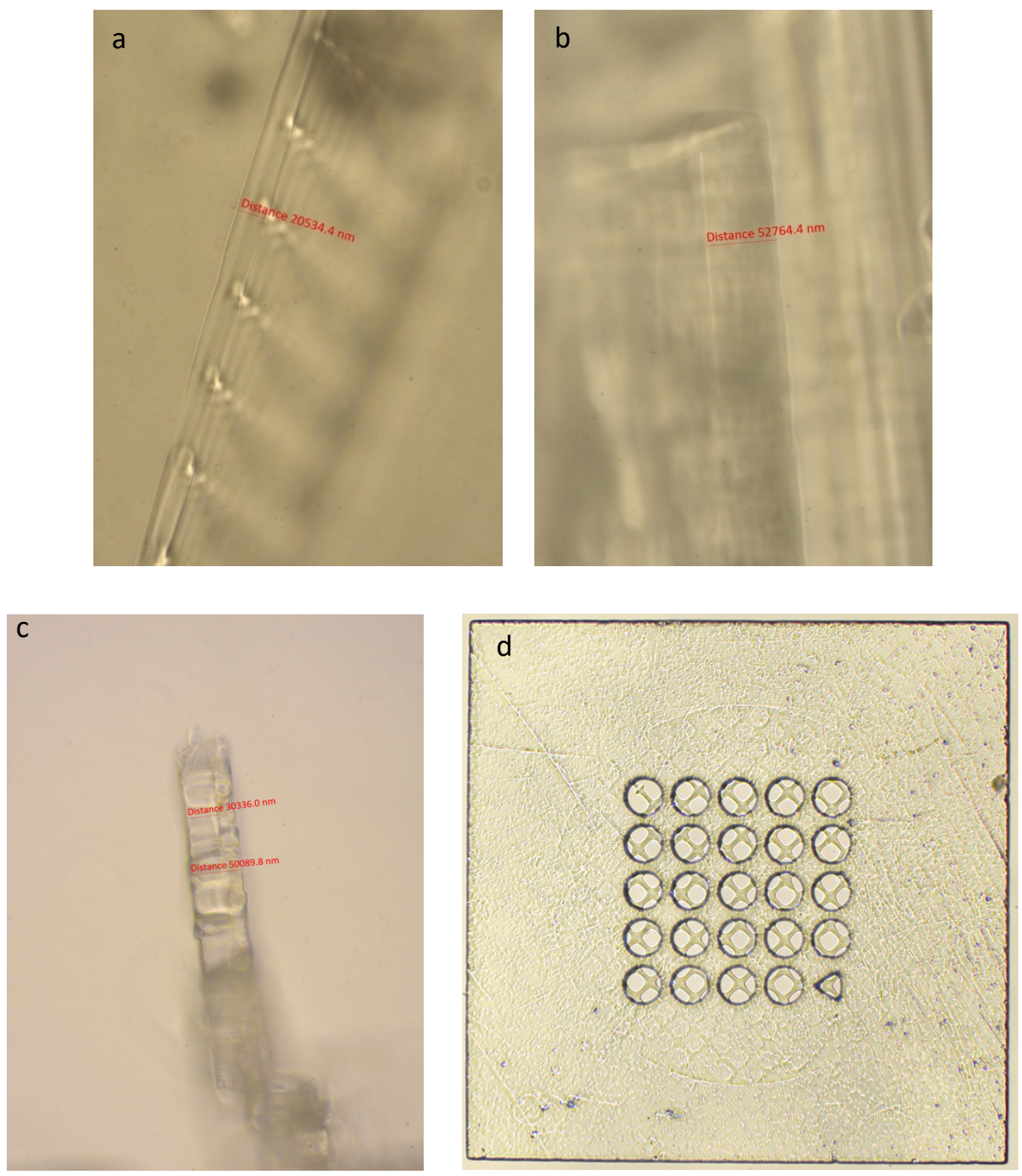

Figure 11. Side view of: (a) Mesh layer with height of $\sim \mathbf{2 1} \mu \mathrm{m}$ made in $\mathbf{2 5} \boldsymbol{\mu \mathrm { m }}$ tall channel. (b) Microwell layer with height of $\sim 53 \mu \mathrm{m}$ made in $60 \mu \mathrm{m}$ tall channel. (c) Well with depth of $\sim 30 \mu \mathrm{m}$. (d) Top view of final microwell array fabricated with an overall length of $1.5 \mathrm{~mm}$, mesh pore size of $50 \mu \mathrm{m}$ and a well diameter of $100 \mu \mathrm{m}$.

The two channel heights were combined into a single step channel to seamlessly produce the microwells in a two-step process enabling a microwell to be made in under a minute. The process is outlined in Figure 12. First, the step channel is filled with a prepolymer solution of $47.5 \%$ PEGDA 700, 47.5\% TMPeTA and 5\% photoinitiator. The flow is stopped, and the mesh layer is 
polymerized in the $25 \mu \mathrm{m}$ tall area of the channel. The flow is resumed to move the mesh layer to the $60 \mu \mathrm{m}$ tall area of the channel and the photomask is changed to match the microwell design. Once aligned, the microwell layer is polymerized over top of the microwell layer. After fabricating both layers, the flow is resumed to push the microwell into the outlet of the channel where it is washed in ethanol and an aqueous solution with 5\% TWEEN 20 and stored in said solution. Figure $11 \mathrm{~d}$ shows the final microwell that is produced using this two-step process. After washing the microwells, the overall length of the microwell is $1.5 \mathrm{~mm}$. This is the limit of the polymerization area because beyond these dimensions the UV power decreases resulting in uneven polymerization.

a
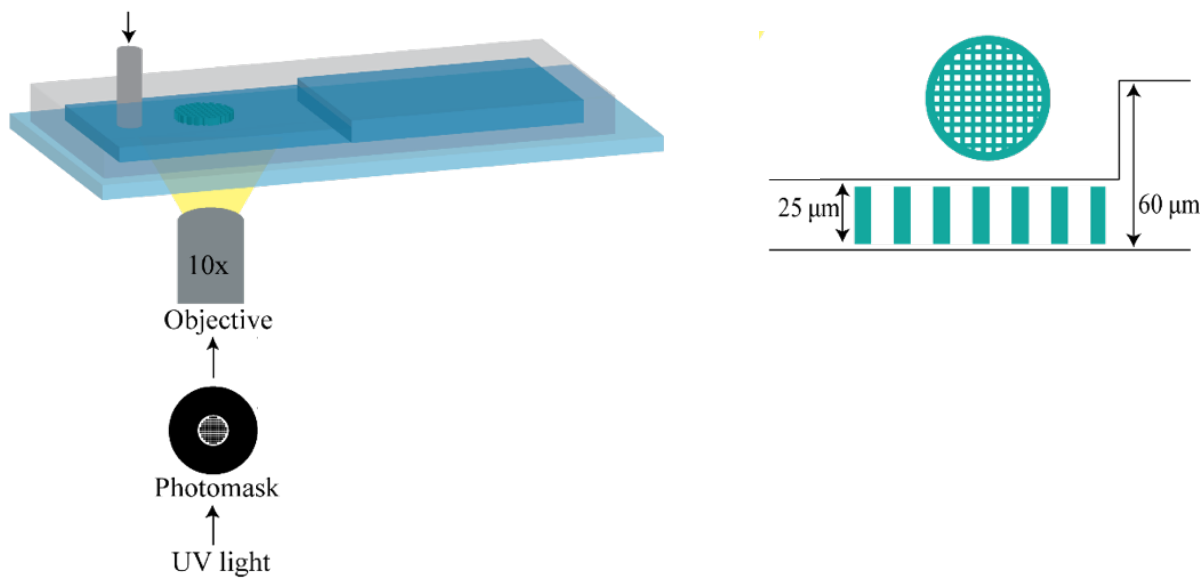

b

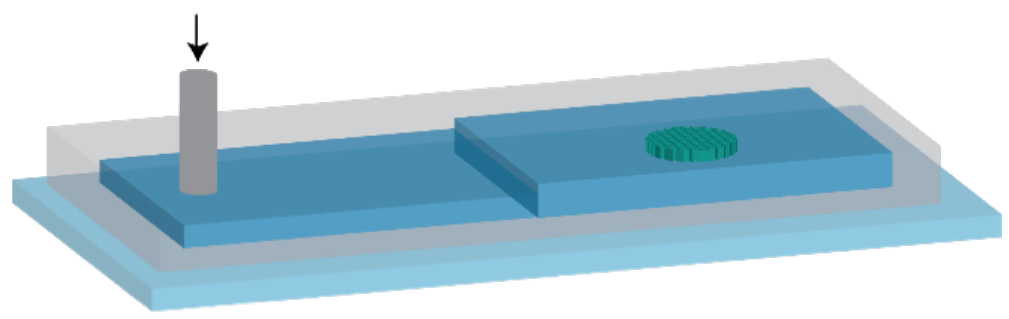




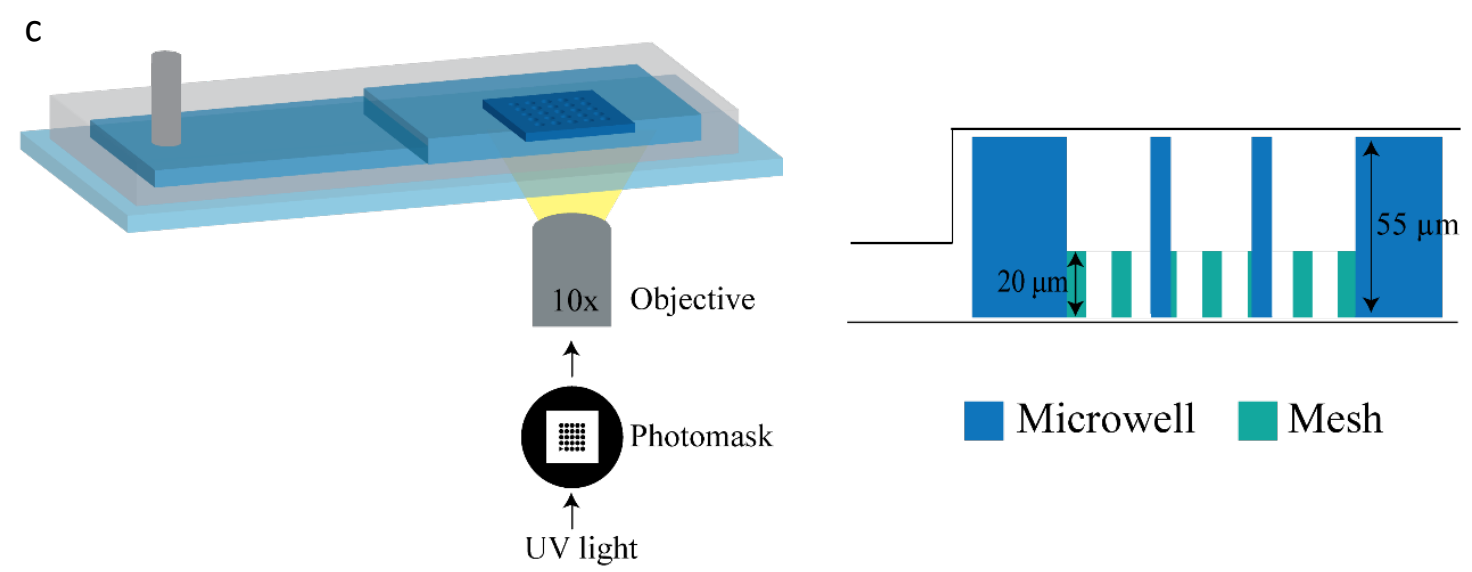

Figure 12. Fabrication schematic of sieved microwells with double layers using slit channel lithography. (a) The mesh layer is fabricated in the $\mathbf{2 5} \mu \mathrm{m}$ tall area of the channel. The side profile showing the cross section of the mesh in the channel. (b) The mesh layer is moved to the $60 \mu \mathrm{m}$ tall area of the channel. (c) The microwell layer is polymerized over top of the mesh layer. The cross section of the channel shows the final pore shape and size of the microwells.

\subsection{Varying Parameters for Polymerization}

The various parameters that are controlled in SCL can be manipulated to attain different sized pores and lengths of microwells. As mentioned, the height and well depth were set based on the double channel that the microwell is installed in. However, the exposure time, photomask or objective can be changed to produce different size microwells.

\subsubsection{Objective}

The objective determines the size of the area that is exposed to light. As mentioned, smaller objectives increase the focal area of the channel, however along the edges of the focal area the light source begins to taper off. Therefore, the overall length is limited to the area that the light source is uniform throughout. A light gradient would result in less polymerization along the edges, creating faded features opposed to the sharp edges that are desired. Experimentally, it was determined that the maximum length for constant polymerization is $1.5 \mathrm{~mm}$ using the $10 \times$ 
objective. Therefore, to make $100 \mu \mathrm{m}$ pores at this objective the array is limited to a $5 \times 5$ array but to make larger arrays with the same pore size, the overall length of the polymerization needs to be increased.

To increase the microwell array, a $5 \times$ objective is used to create a larger projection area. With this objective everything is double in size from the $10 \times$ objective. Therefore, by using the same photomasks as at $10 \times$, the dimension of the microwells will be doubled at $5 \times$. For example, a pair of photomasks used to create a well diameter of $50 \mu \mathrm{m}$, mesh pore length of $25 \mu \mathrm{m}$ and overall length of $1.5 \mathrm{~mm}$ at $10 \times$ fabricates a $8 \times 8$ array of microwells with a diameter of $100 \mu \mathrm{m}, 50 \mu \mathrm{m}$ mesh pore length and an overall length of $3 \mathrm{~mm}$ with at $5 \times$ (Figure 13). Although, the larger area disperses the light energy over a larger area and a longer exposure time is required compared to the 10x. Long UV light exposures can result in deformation of the shape. The flow in the channel does not completely stop at any point, it only slows, so polymerization would occur as the solution is moving. To prevent this, violet light is used instead of UV light since it has a longer wavelength, in addition to a different photoinitiator which is reactive to violet light.

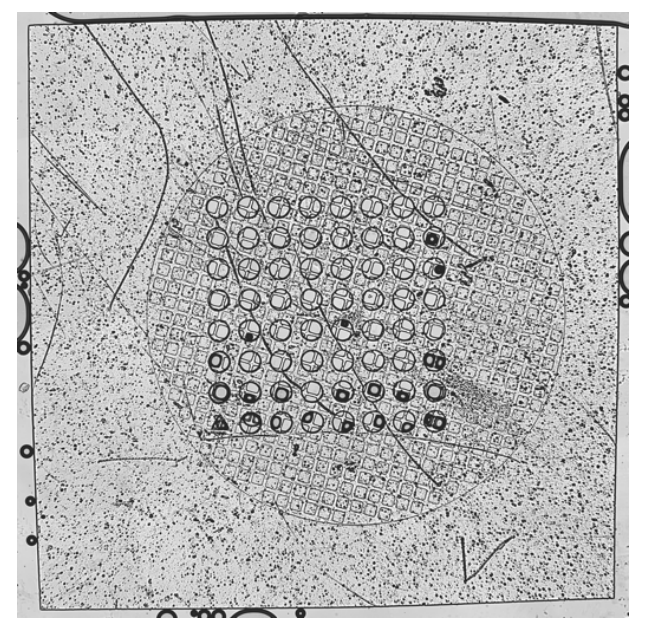

Figure 13. Microwells with an overall length of $3 \mathrm{~mm}, 100 \mu \mathrm{m}$ well diameter and $50 \mu \mathrm{m}$ pore length. 


\subsubsection{Photomasks}

Changing the photomask has the most noticeable effect on the pore shape and size. The ability to easily change the photomasks to create different sized wells and pores makes this fabrication method very versatile for a wide range of particle sizes. By customizing microwells, it is possible to capture both single and multiple particles in a variety of shapes. Well diameters have been made in diameters from 30 to $100 \mu \mathrm{m}$ (Figure 14a), in addition to being made into various shapes, including triangular, square, pentagonal and hexagonal (Figure 14b). When changing the well diameter, the mesh length is also changed to correspond.
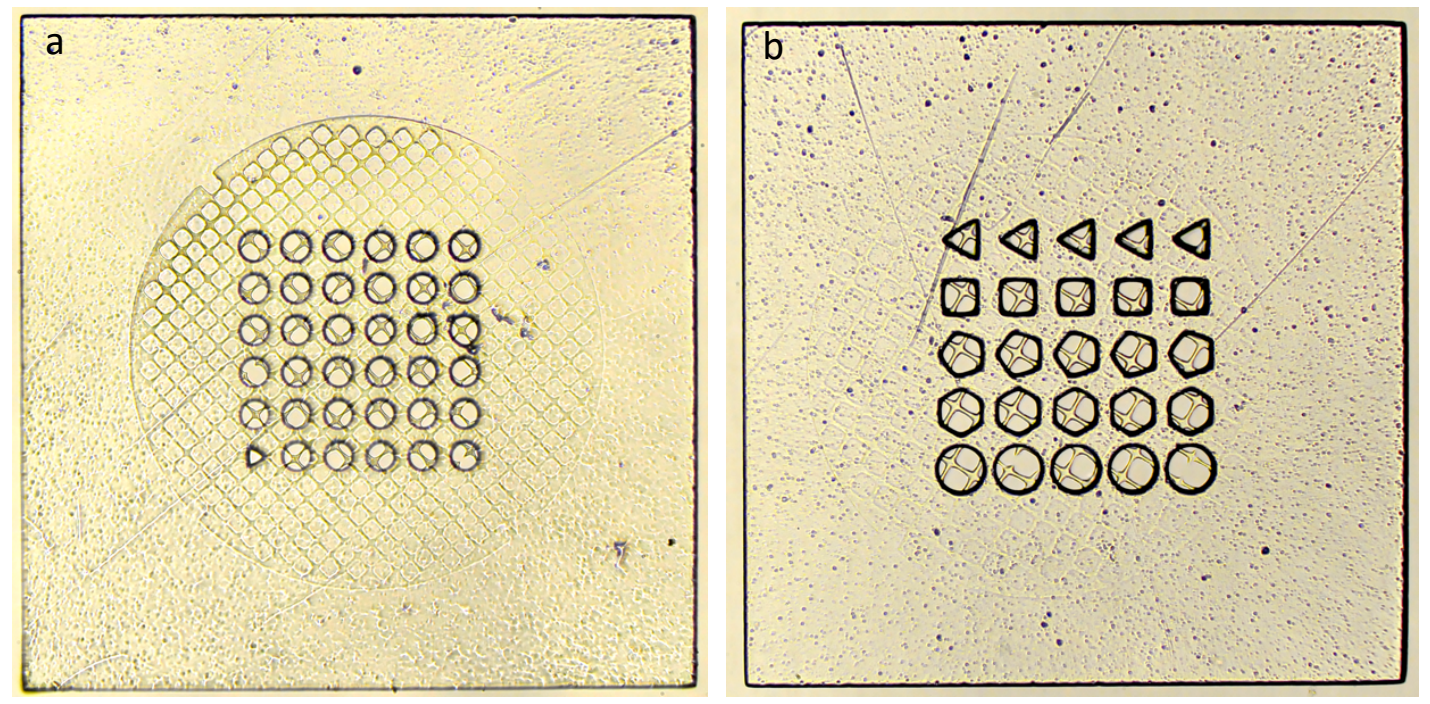

Figure 14. Microwells with different well diameters and shapes. (a) Microwells with an overall length of $1.5 \mathrm{~mm}, 60 \mu \mathrm{m}$ well diameter and $30 \mu \mathrm{m}$ pore length. (c) Microwells with an overall length of 1.5 $\mathrm{mm}, 100 \mu \mathrm{m}$ well diameter and $40 \mu \mathrm{m}$ pore length.

The mesh pore size can be made into various feature sizes based on the photomask, creating a range of pore lengths from 20 to $60 \mu \mathrm{m}$ (Figure 15a,b,d), in addition, to being used to define the spacing between the pores (Figure 15c). The spacing is important since narrower spacing can easily break during the washing and installing process but overly wide spacing can close off a 
larger portion of the bottom of the wells which would reduce hydrodynamic flow through the wells. This approach requires an extensive number of photomasks for each size and spacing, in addition, the smallest pore length possible is $20 \mu \mathrm{m}$. Photomasks with smaller pore lengths can be made but using the same conditions for UV light exposure results in closed pores. In order to create smaller pores, a reduced exposure time is necessary.

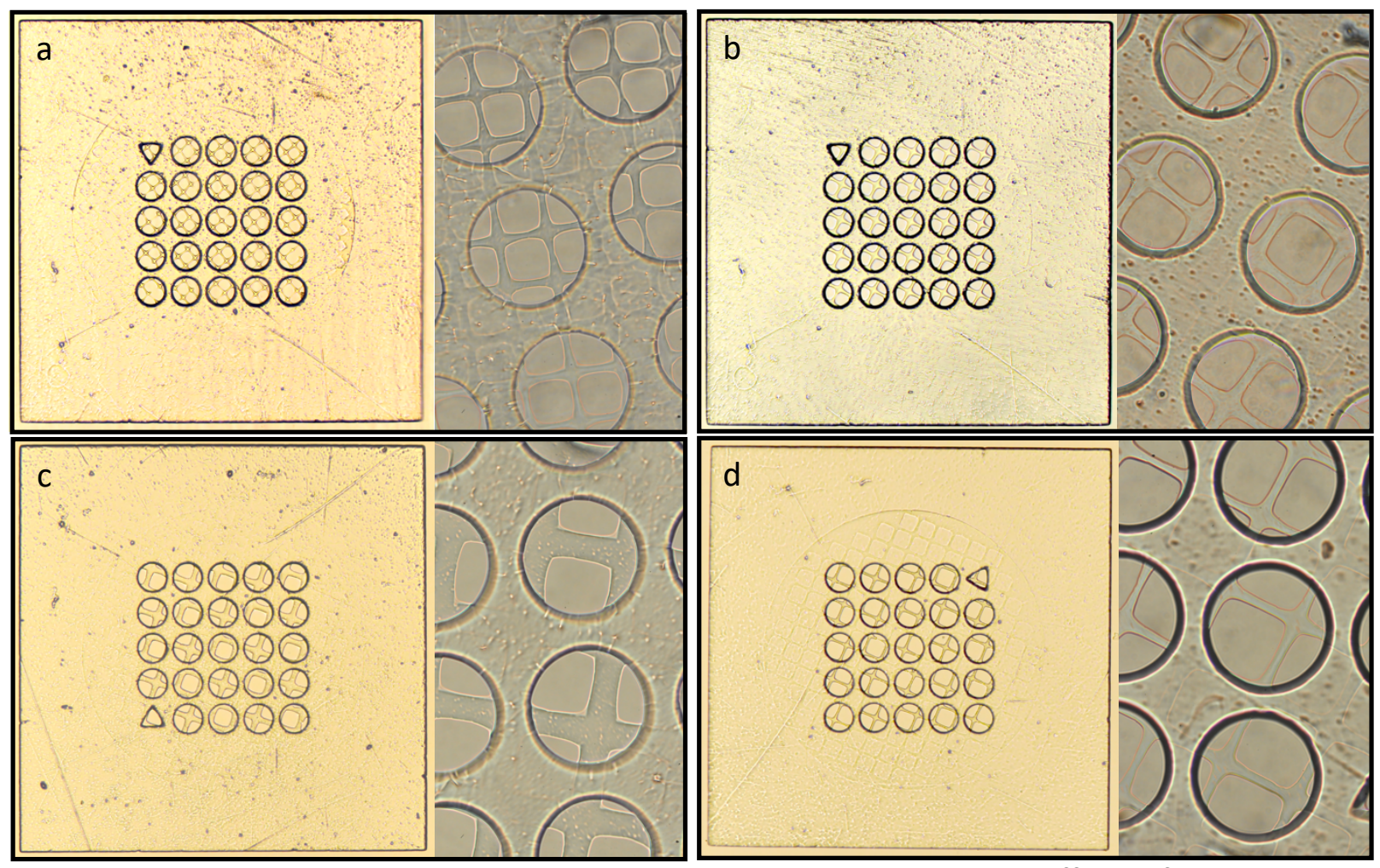

Figure 15. Microwells with $100 \mu \mathrm{m}$ wells and mesh sizes controlled using different feature-sized photomasks, resulting in pore widths of: (a) $30 \mu \mathrm{m}$, (b) $40 \mu \mathrm{m}$, (c) $50 \mu \mathrm{m}$ with a $20 \mu \mathrm{m}$ spacing, and (d) $60 \mu \mathrm{m}$.

\subsubsection{UV Exposure Time}

To create smaller pores sizes and limit the number of photomasks required, the exposure dose can be adjusted. Using a single photomask, it is possible to reduce the pore size with increased exposure time because free radical diffusion starts occurs outside the projected area. 
Alternatively, reducing the exposure time results in less cross-linking making larger pores in addition to a more fragile structure. Using a photomasks that was designed to fabricate $40 \mu \mathrm{m}$ long pores with an exposure time of $200 \mathrm{~ms}$, it is possible to create larger pores up to $43 \mu \mathrm{m}$ in length with a shorter exposure time of 150 ms (Figure 16a). However, any exposure time less than 150 ms results in low crosslinking density, leading to a fragile spacer and easy breakage. If the exposure time is increased over $200 \mathrm{~ms}$, the pore length significantly decreases until it closes at 600 ms (Figure 16c). The graph in Figure 16d shows how the pore change with the change in exposure time, with a constant UV intensity of $80 \%$.
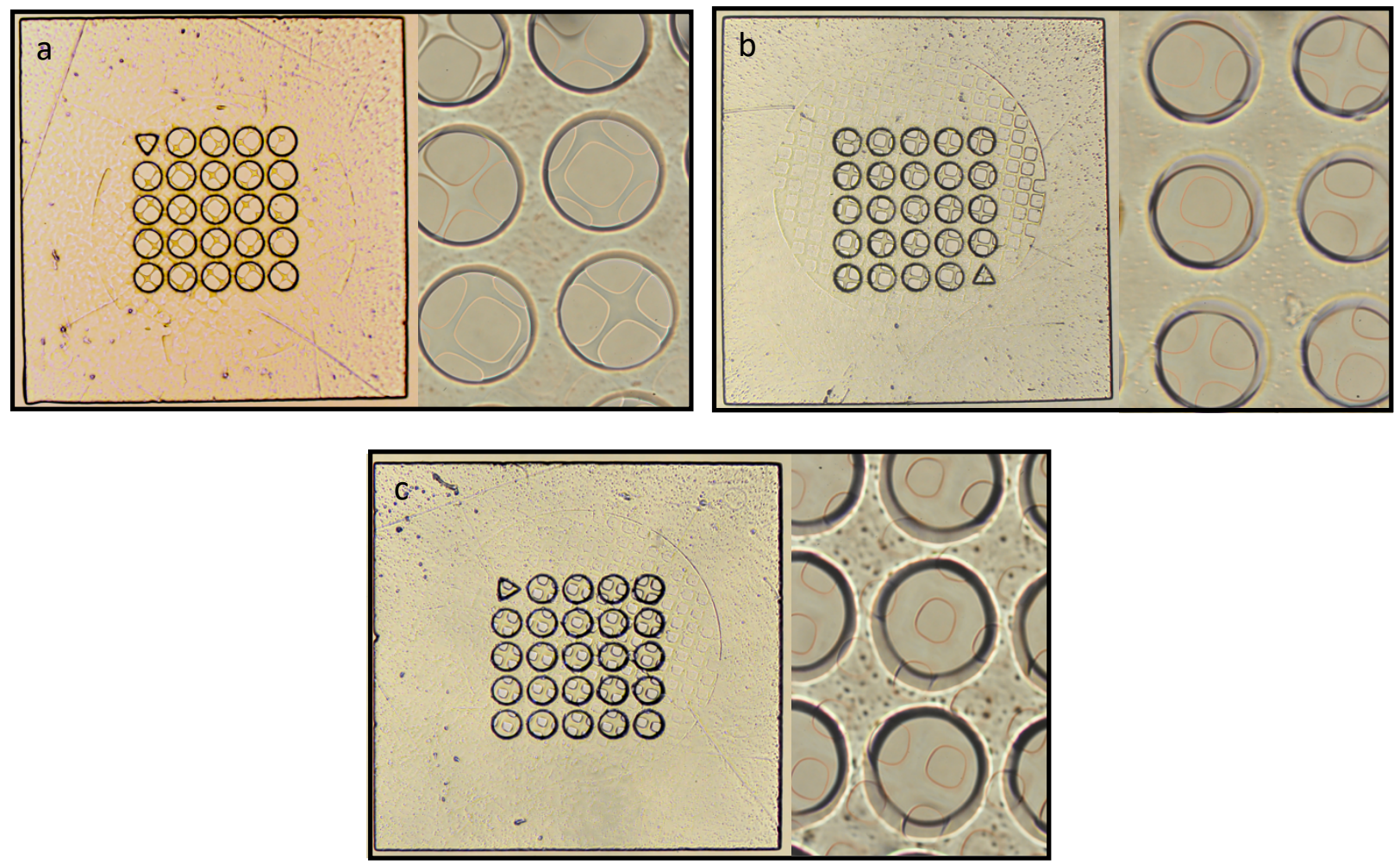


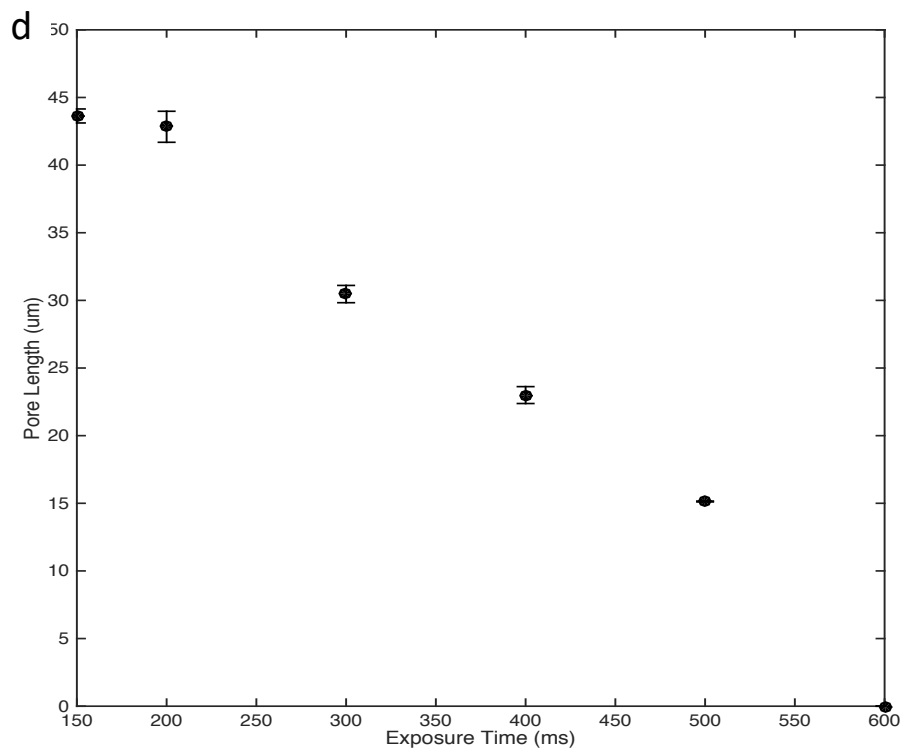

Figure 16. Mesh pore control with UV dose. Bright field images of microwells with $100 \mu \mathrm{m}$ wells and mesh sizes controlled by changing the exposure time, using times of: (b) $150 \mathrm{~ms}$, (c) $250 \mathrm{~ms}$, and (d) $500 \mathrm{~ms}$. (d) Plot of the size of mesh pore reduction with an increase exposure time, resulting in completely closed pores at $600 \mathrm{~ms}$.

\subsection{Capturing Particles in Sieved Microwells}

The microwells were installed in a microfluidic double channel with a cross-flow arrangement to capture particles. The cross-flow increases the pressure drop across the microwell surface, in addition to decreasing fouling, which in this case is the accumulation of particles over the microwell. To install the microwell two techniques were used, one installs the microwell into a preassembled channel through the top channel outlet, and the other assembles the channel with the microwell already installed.

As seen in Figure 17, the assembled microfluidic channel is made by aligning two channels perpendicularly to each other to create an intersection for cross-flow. The vertical channel is the top channel, which the particles are flowed through. The top $1 \mathrm{~mm}$ inlet is used for shielding flow which runs along the edges of the channel to prevent particles from being pulled towards the 
side of the microwell. The inlet below it is used to introduce the particles down the center of the channel. The outlet of the top channel is used to install the microwell, while the other two outlets are connected to the bottom channel which allows flow through the bottom channel.

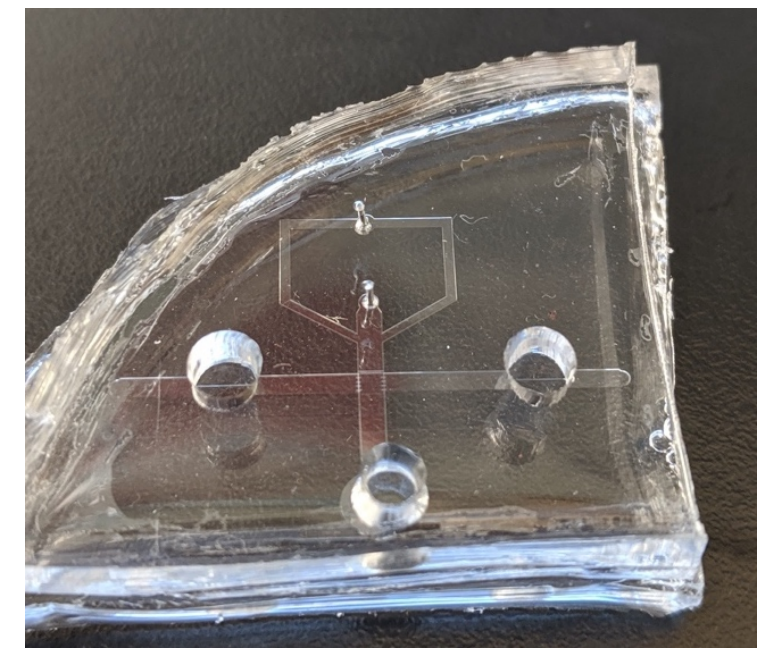

Figure 17. Assembled cross-flow microfluidic channel.

\subsubsection{Microwell and Particle Size Selection}

The seating area for the microwell is a $1.5 \mathrm{~mm}$ square recessed $60 \mu \mathrm{m}$ into the bottom channel. The height of the microwell is designed to be slightly lower than the bottom of the top channel to prevent shear stress from washing it out since it is not physically attached to the channel (Figure 18). Therefore, the microwells were made to be $55 \mu \mathrm{m}$ in height. Additionally, the microwell is made to fit perfectly into the bottom slot to prevent leaking along the edges of the microwell. Gaps along the edges of the microwell provide an alternate route for flow of particles which can decreases the trapping efficiency. Shielding flow is added to prevent this, however a well-fitting microwell is important to limit the gap along the edges. Therefore, the microwell is fabricated to be close to $1500 \mu \mathrm{m}$. 
Since the microwells are made out of hydrogels, the swelling of the material needs to be considered when fabricating the array to be $\sim 1500 \mu \mathrm{m}$. The prepolymer solution used to make the microwells (47.5\% PEG-DA, 47.5\% TMPeTA, 5\% photoiniator) has an average swelling ratio of 4\% in the 5\% Tween 20 solution. A different prepolymer solution, for example 95\% PEG-DA 700 and $5 \%$ photoinitiator, has a higher swelling ratio of $10 \%$ in the same solution because of the different material properties. Since the composition of the prepolymer solution has such a significant effect on the swelling ratio, the solution is be mixed prior to each use. If not, the solution can separate which can result in unexpected swelling ratios or a notable difference in integrity of the material. The microwell array is polymerized to be $\sim 1440 \mu \mathrm{m}$ in length and expands to a final length of $\sim 1500 \mu \mathrm{m}$. When designing the photomasks for the microwells a smaller photomask is designed so that the microwell array swells to the desired size.

Lastly, the particle and well size were selected. The height of the particles was limited to $~ 30 \mu \mathrm{m}$ to prevent shear stress from washing them out during operation, since the well depth was fixed at $\sim 35 \mu \mathrm{mm}$. In terms of the diameter of the particles, both smaller and larger particles were tried. Initially, smaller diameter particles with diameters $40-50 \mu \mathrm{m}$ were through to be ideal since they would be easier to flow through the channel. However, during operation the smaller particles were easily jammed into any gaps along the edge of the device which would easily dislodge the array. Also, at this size the particles were able to turn sideways in the channel causing multiple particles to be captured within the same well. Since single particle capture was the aim of the device, a larger particle diameter of $90 \mu \mathrm{m}$ was selected to prevent rotation in the channel. When using larger particles, the microwell was displaced less but multiple particles per 
well would still occur but with less frequency. Therefore, the final particle dimensions used were $90 \mu \mathrm{m}$ diameter particles with a $30 \mu \mathrm{m}$ height. To obtain single particle trapping using these particles, the well diameter used was $100 \mu \mathrm{m}$ in diameter with a pore length of $50 \mu \mathrm{m}$. These dimensions are used throughout the remainder of the experiments.
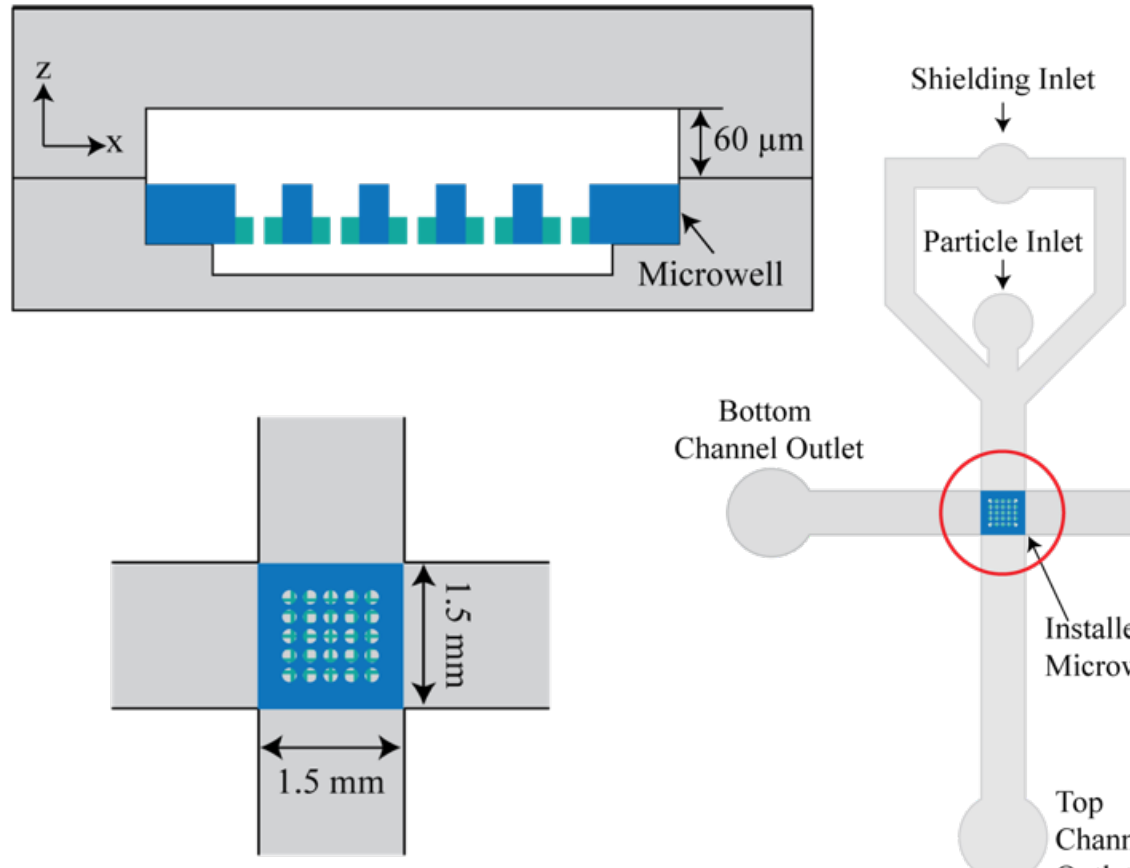

Bottom Channel Outlet

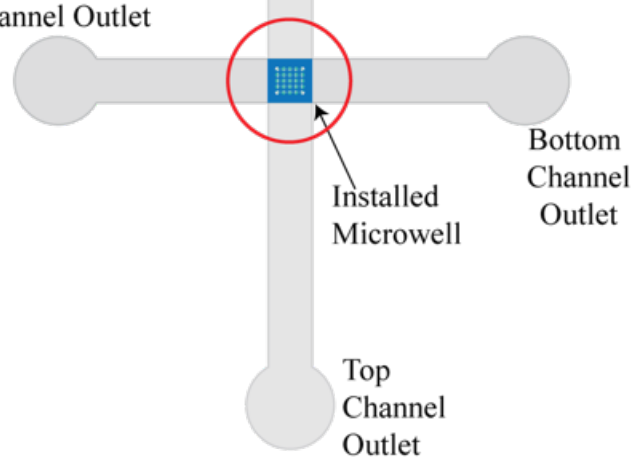

Figure 18. Installation of microwell using $60 \mu \mathrm{m}$ tall top channel

\subsubsection{Assembling and Using a 60 um Tall Top Channel}

Initially, a top channel with a width of $1500 \mu \mathrm{m}$ and height of $60 \mu \mathrm{m}$ was used for the double channel (Figure 18). The benefit of this channel design is that the microwell array can be installed and removed repeatedly. This allows for an array loaded with particles to be removed, in addition to being able to use the channel multiple times with different microwells. Assembling this 
channel can be challenging since the alignment of the top and bottom channel needs to be perfect to ensure that the microwell fits perfectly into cross-section which can be challenging on such a small scale. Also, the channels can be easily deformed when removing them from the master mold if the PDMS is not cured enough.

To install the microwell in this set up, the outlet of the top channel needs to be tall enough to accommodate the height of the microwell which is why the top channel was designed to be 60 $\mu \mathrm{m}$ in height and $1500 \mu \mathrm{m}$ in width. After aligning and curing the two PDMS channels, the microwell can be introduced through the outlet of the top channel. The microwell is pipetted into the outlet and based on the orientation of the triangular pore, the microwell is flipped to determine the orientation of the wells (Figure 19a). It is important to ensure that the wells are facing upwards to capture the particles. During this step a large number of microwells can be broken if the structure of the microwell which is why the composition of the prepolymer solution is important. The TMPeTA 438 provides structure to the microwell while the PEG-DA 700 prevents it from becoming overly rigid. A syringe is used to push the microwell into the entrance of the top channel and a little solution is used to flow the microwell into the intersection (Figure 19b). Once the microwell is docked it is supported along the bottom edges by a series of posts and is held in place by the hydrodynamic pressure during operation (Figure 19c).

Throughout this process it is important that no air bubbles are in the channel, therefore when preparing any syringes, the air push out prior to inserting into the channel and the syringe pumps are running prior to inserting into the channel. Air bubbles in the channel can cause blockages along the channel, preventing particles from flowing smoothly or if the bubble is located under 
the microwell seating area, it can push the microwell upwards and easily dislodge it from the intersection.
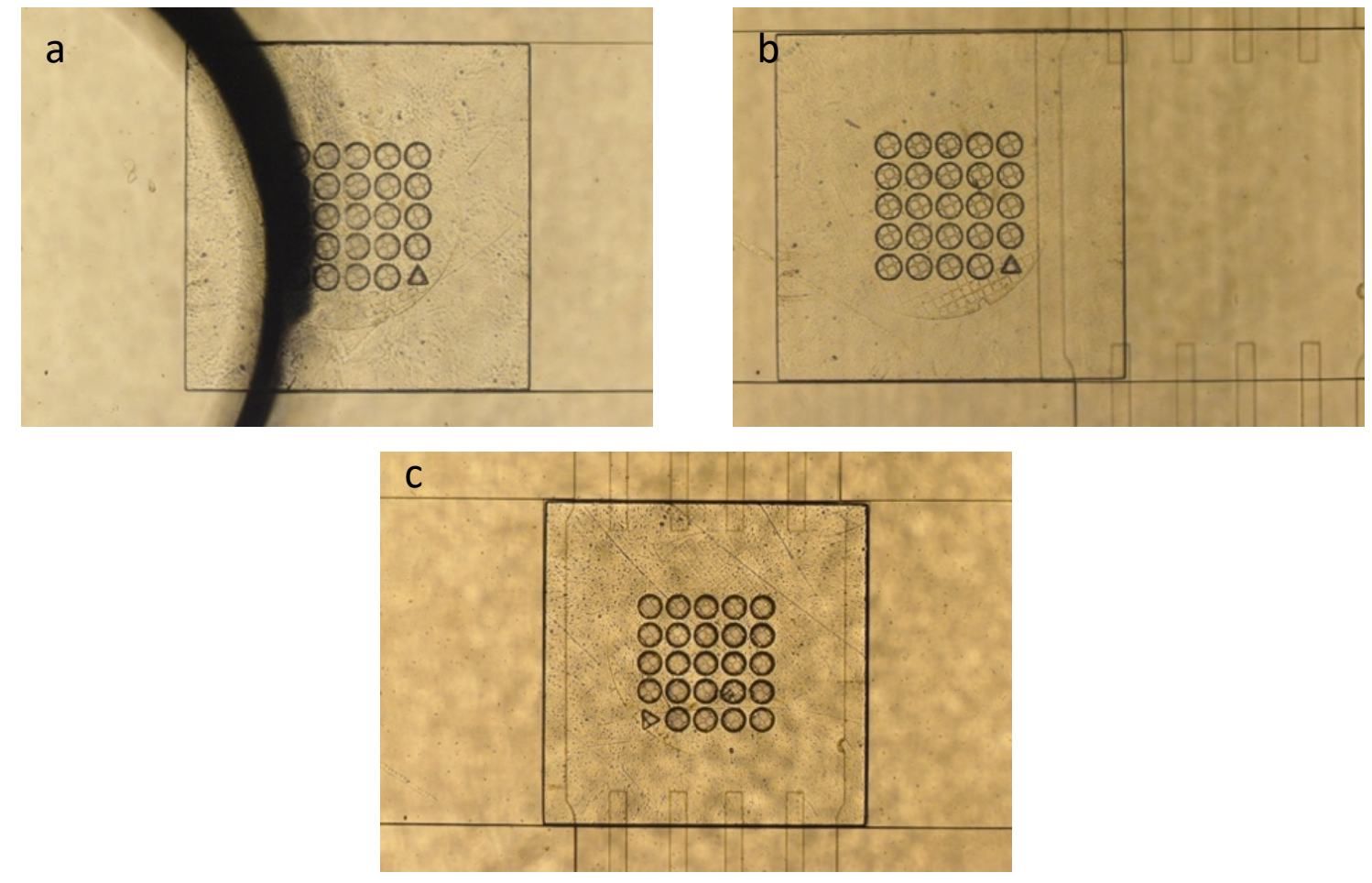

Figure 19. Installing a microwell through the outlet of a $60 \mu \mathrm{m}$-tall top channel. (a) Microwell is inserted into the outlet. (b) Microwell flows down channel. (c) Microwell is docked into seating area.

Finally, the microwell capture ability is assessed in this crossflow configuration. Cylindrical particles, with a density of approximately $10^{4}$ particles $/ \mathrm{mL}$, are flowed through the channel. The particles are flowed along the top channel with flow rates of $50-100 \mu \mathrm{L} / \mathrm{min}$. Shielding flow rates, which flow along the edge of the microwell to deter the particle stream from flowing that way, were used at the same flow rate as the particles. With this range of flow rate, an approximate $52 \%$ well occupancy is achieved (Figure 20). The poor capture efficiency could be caused by the height of the top channel. The top channel was double the height of the particles, so the particles were not close enough to the wells to be pulled in by the hydrodynamic flow 
through the wells. Also, numerous particles were accumulating over the surface of the wells or along the edge of the channels preventing a continuous flow of particles over the wells. Higher flow rates could be used to resolve this but the microwell array was easily displaced at high flow rates making it hard to operate.

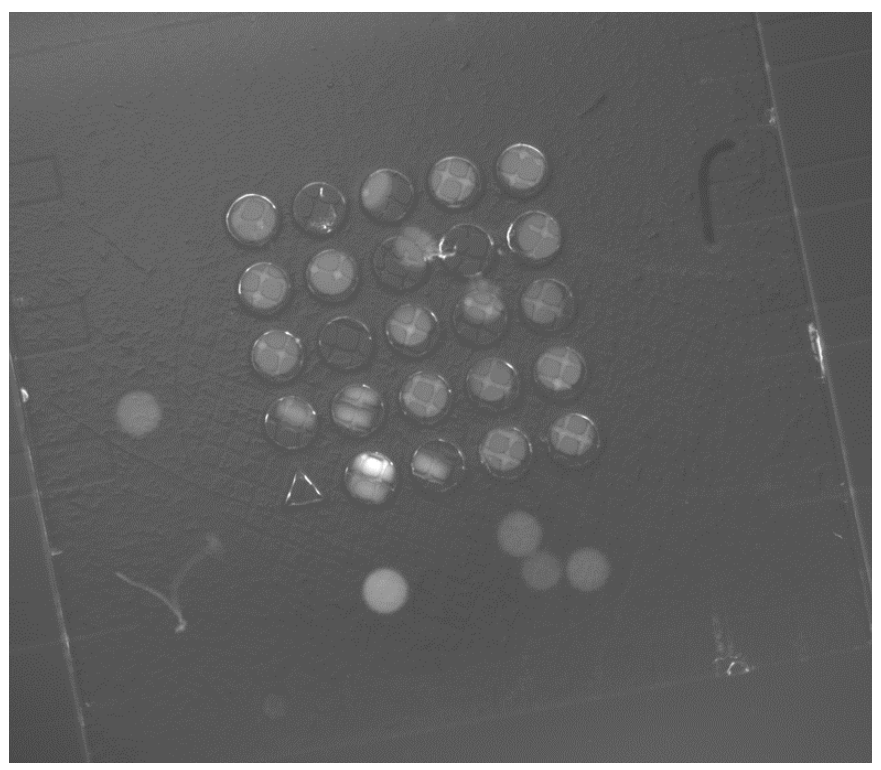

Figure 20. Captured particles in $100 \mu \mathrm{m}$ diameter wells using a flow rate of $50 \mu \mathrm{L} / \mathrm{min}$

\subsubsection{Assembling and Using a 30 um tall Top Channel}

To reduce the top channel height and better secure microwells for use at higher flow rates, a new assembly route was developed for the microwell array installation ( Figure 21). In this route, a top channel with a height of $30 \mu \mathrm{m}$ and a width of $1300 \mu \mathrm{m}$ is used to bring particles in closer contact with the microwells, however, using this method the microwell can no longer be installed through the top channel. Instead the channel is assembled around the microwells. Using the same bottom channel as previously, a microwell array is pipetted onto the seating area of the bottom channel and oriented so the wells are facing upwards. The bottom channel, with the 
microwells, is placed in a desiccator to remove any excess liquid. Both the top and bottom channels are then plasma treated and bonded together. The slimmer top channel made alignment easier since perfect alignment was no longer necessary. In this configuration, the microwell is physically constrained by the top channel since overlapping the top channel over the microwell prevents the microwell from sliding out of the seating area, allowing it to withstand higher flow rates. After assembling the channel, a 5\% Tween-20 solution is introduced into the channel ensuring the microwells swell back to its' original width of $1500 \mu \mathrm{m}$ ( Figure 21).
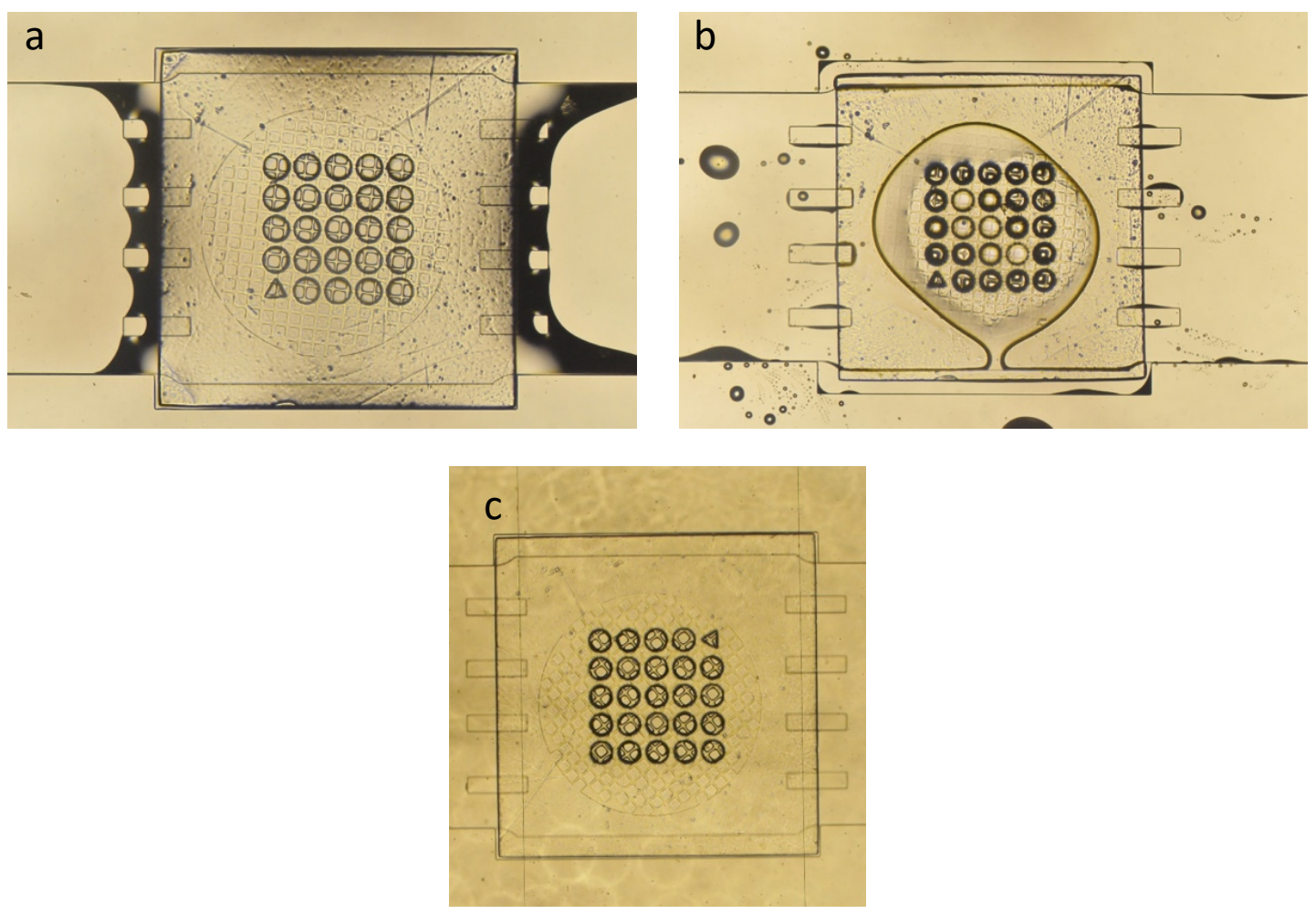

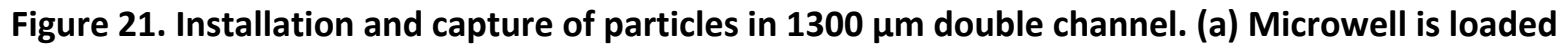
onto bottom channel (b) Microwell is dehydrated. (c) Final assembled channel.

First, the same flow range of $50-100 \mu \mathrm{L} / \mathrm{min}$ was applied using this narrower top channel resulting in an increased well occupancy of up to $73 \%$ (Figure 22). Nevertheless, a large number of particles were still accumulating over the surface of the microwell as seen previously. These 
results indicate that a lower top channel helped in increasing the occupancy, however a higher flow rate is needed to clear the surface of the microwells and promote trapping.
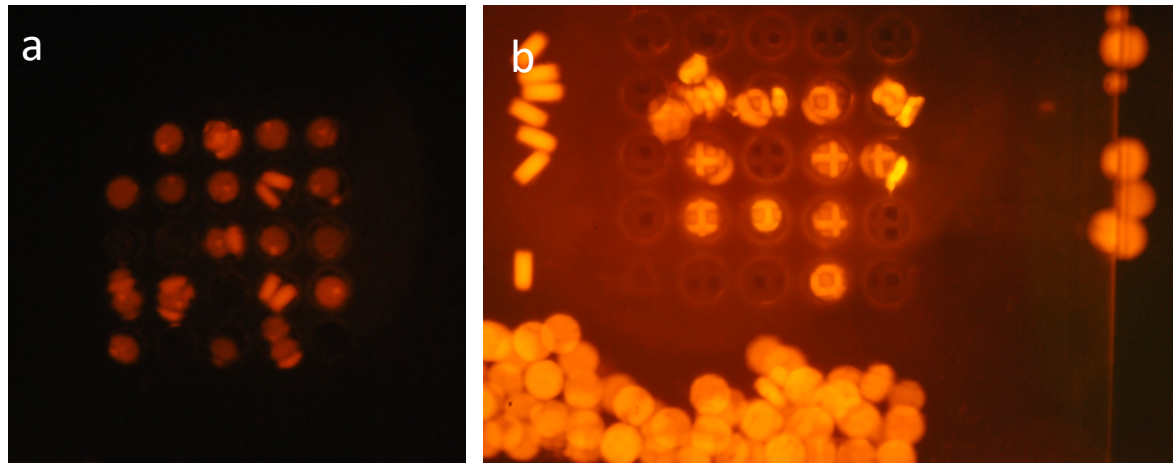

Figure 22. Fluorescent images of the particles captured using a flow rate of $50 \mu \mathrm{L} / \mathrm{min}$. (a) Resulting well occupancy of approximately $73 \%$ (b) Numerous particles scatter along the surface.

Using the maximum flow rate of the syringe pump (purging mode), approximately $9 \mathrm{~mL} / \mathrm{min}$, for one second intervals provided the best results. With each subsequent flow of particles at this high flow rate the array was filled with more particles each time. After seven $1 \mathrm{~s}$ intervals at the maximum flow, the well occupancy of the microwells was $87 \%$. Figure 23 shows a time lapse of the process with the final image being at the $7 \mathrm{~s}$ point where the maximum occupancy is achieved. The particles stay in place with each interval of high flow which indicates that they are locked in place by the hydrodynamic flow. The high flow rate is possible using this arrangement because the narrower top channel sandwiches the microwells in place. Additionally, the ability to fill a microwell array in under $10 \mathrm{~s}$ is a significant reduction in the time compared to other microwell arrangements which usually take at least $1 \mathrm{~min}$ to fill. 

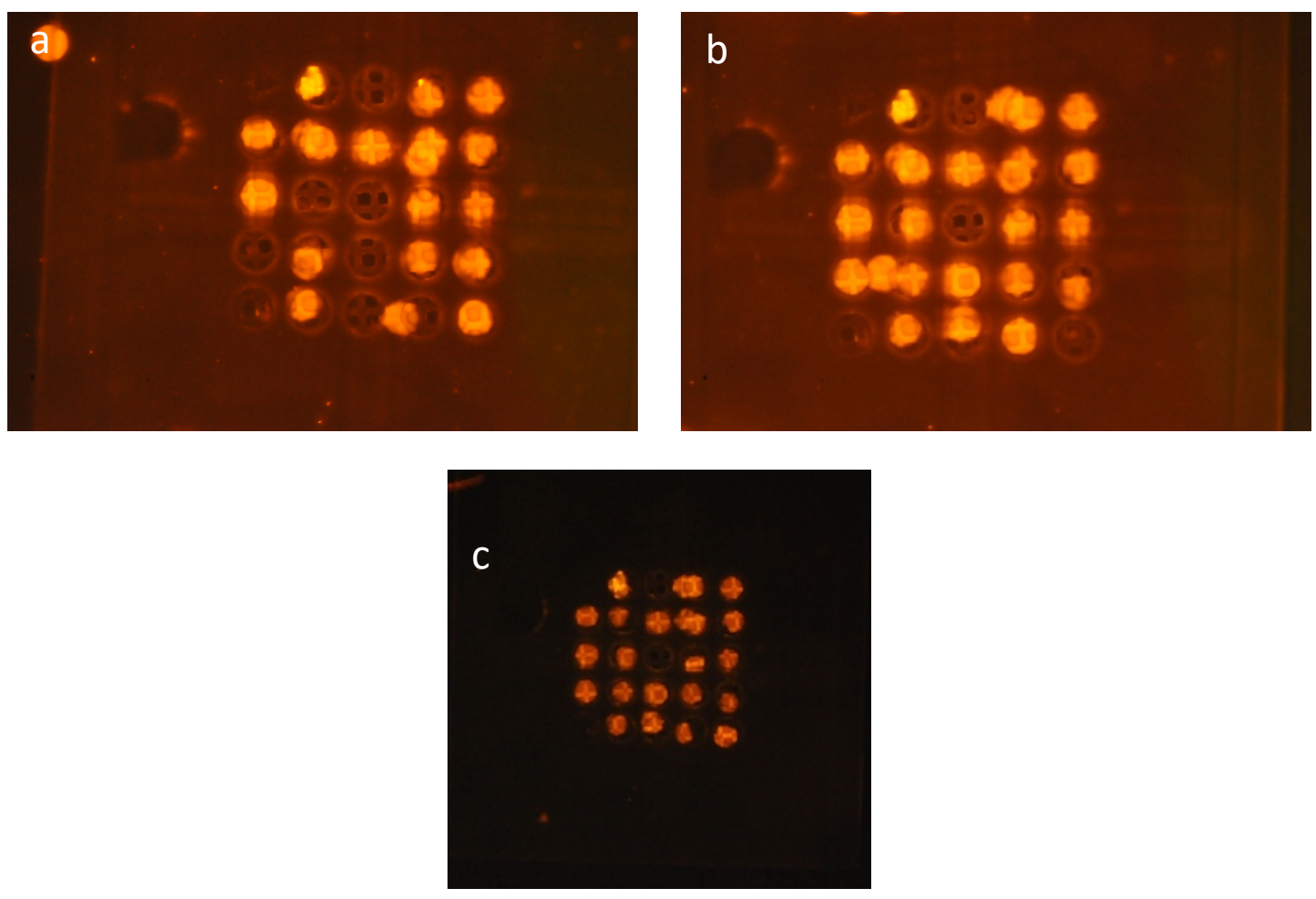

Figure 23. Fluorescent images of time lapse of particles captured using a flow rate of $\sim 9 \mathrm{~mL} / \mathrm{min}$. (a)Well occupancy after $4 \mathrm{~s}$. (b) Well occupancy after $6 \mathrm{~s}$ (c) After $7 \mathrm{~s}$ a well occupancy of $87 \%$ is achieved.

The capture time is greatly reduced compared to other microwell trapping mechanisms. Of the methods listed in Table 1 and Table 2, using negative pressure in a static porous microwells resulted in the shortest capture time of approximately 1 minute to fill an array [28]. The method demonstrated here is able to fill an array of wells in $12 \%$ of the time, significantly minimizing the required capture time. 


\section{Conclusions and Recommendations}

\subsection{Conclusions}

A sieved microwell array was fabricated in a simple two-step process using slit channel lithography which enables rapid fabrication of microwells. This fabrication method allows for a wide range of well and mesh sizes and shapes to be easily made by changing the photomask, objective or UV exposure time at constant power. Installing the microwells in a cross-flow microfluidic device significantly improves the capture rate of particles into the wells using hydrodynamic force through the open pores at the bottom of the wells. Furthermore, the installation methods of the microwells into the microfluidic device demonstrates the versatility of this arrangement. One method permits the easy retrieval of the microwell from the channel, which can facilitate isolation of specific cells or particles for further study, while the other allows for higher flow rates to be used which significantly reduces the capture time to under $10 \mathrm{~s}$ and occupancy of the wells to $87 \%$.

\subsection{Recommendations}

The work completed demonstrates the capture ability of sieved microwells, however, further study into the various applications sf sieved microwells are necessary. These types of microwells can be beneficial to biological studies through applications in single cell trapping.

By applying sieved microwells to cell capture and single cell analysis it would be possible to capture individual cells quickly. Further study into the comprehensive study of optimal flow conditions with various sizes of well and mesh used should be done. The design of these wells 
improves cellular studies compared to traditional microwells by providing uniform nutrient dispersion throughout the wells, as opposed to relying of diffusion in conventional wells, in addition to allowing for seamless aqueous media changes in this device. Additionally, the shared media would allow for cell to cell communication which is not possible in a traditional microwell platform.

Additionally, the ability to remove the particles from the microwells has yet to be demonstrated. Cell isolation for further studies is an important feature for microwells, therefore the ability to withdraw a loaded array from the microfluidic channel should be considered. Although one channel allows for the withdrawal of the array, it does not provide sufficient trapping so further study into the optimal trapping in this arrangement would be an asset. 


\section{References}

[1] D. Gao, F. Jin, M. Zhou, and Y. Jiang, "Recent advances in single cell manipulation and biochemical analysis on microfluidics," Analyst, vol. 144, no. 3, pp. 766-781, 2019.

[2] S. H. Kim, G. H. Lee, and J. Y. Park, "Microwell fabrication methods and applications for cellular studies," Biomedical Engineering Letters, vol. 3, no. 3. Springer Verlag, pp. 131137, 2013.

[3] H. Yin and D. Marshall, "Microfluidics for single cell analysis," Current Opinion in Biotechnology, vol. 23, no. 1. pp. 110-119, Feb-2012.

[4] S. Lindström and H. Andersson-Svahn, "Miniaturization of biological assays - Overview on microwell devices for single-cell analyses," Biochimica et Biophysica Acta, vol. 1810, no. 3. pp. 308-316, Mar-2011.

[5] H. Andersson and A. Van Den Berg, "Microtechnologies and nanotechnologies for singlecell analysis," Current Opinion in Biotechnology, vol. 15, no. 1. Elsevier Ltd, pp. 44-49, 2004.

[6] R. M. Johann, "Cell trapping in microfluidic chips," Anal. Bioanal. Chem., vol. 385, no. 3, pp. 408-412, Jun. 2006.

[7] T. Braschler, N. Demierre, E. Nascimento, T. Silva, A. G. Oliva, and P. Renaud, "Continuous separation of cells by balanced dielectrophoretic forces at multiple frequencies," Lab Chip, vol. 8, no. 2, pp. 280-286, 2008.

[8] A. Keloth, O. Anderson, D. Risbridger, and L. Paterson, "Single cell isolation using optical tweezers," Micromachines, vol. 9, no. 9, 2018.

[9] T. Kimura, Y. Sato, F. Kimura, M. Iwasaka, and S. Ueno, "Micropatterning of cells using modulated magnetic fields," Langmuir, vol. 21, no. 3, pp. 830-832, 2005.

[10] K. Cheung, S. Gawad, and P. Renaud, "Impedance spectroscopy flow cytometry: On-chip label-free cell differentiation," Cytom. Part A, vol. 65, no. 2, pp. 124-132, 2005.

[11] K. Galler, K. Bräutigam, K. Bräutigam, C. Große, J. Popp, and U. Neugebauer, "Making a big thing of a small cell - recent advances in single cell analysis," Analyst, vol. 139, no. 6, pp. 1237-1273, Feb. 2014.

[12] N. T. Huang, Y. J. Hwong, and R. L. Lai, "A microfluidic microwell device for immunomagnetic single-cell trapping," Microfluid. Nanofluidics, vol. 22, no. 2, pp. 1-8, Feb. 2018.

[13] A. Grünberger, W. Wiechert, and D. Kohlheyer, "Single-cell microfluidics: Opportunity for bioprocess development," Current Opinion in Biotechnology, vol. 29, no. 1. Elsevier Ltd, pp. 15-23, 2014.

[14] D. K. Wood, D. M. Weingeist, S. N. Bhatia, and B. P. Engelward, "Single cell trapping and DNA damage analysis using microwell arrays," in Proceedings of the National Academy of Sciences of the United States of America, 2010, vol. 107, no. 22, pp. 10008-10013. 
[15] J. Doh, M. Kim, and M. F. Krummel, "Cell-laden microwells for the study of multicellularity in lymphocyte fate decisions," Biomaterials, vol. 31, no. 12, pp. 3422-3428, Apr. 2010.

[16] J. Yuan and P. A. Sims, "An Automated Microwell Platform for Large-Scale Single Cell RNASeq," Sci. Rep., vol. 6, Sep. 2016.

[17] L. Zhang et al., "Agarose-based microwell array chip for high-throughput screening of functional microorganisms," Talanta, vol. 191, no. June 2018, pp. 342-349, 2019.

[18] J. L. Ng, Y. Shin, and S. Chung, "Microfluidic platforms for the study of cancer metastasis," Biomed. Eng. Lett., vol. 2, no. 2, pp. 72-77, 2012.

[19] W. Liu et al., "One step DNA amplification of mammalian cells in picoliter microwell arrays," RSC Adv., vol. 9, no. 5, pp. 2865-2869, 2019.

[20] A. B. Bernard, C.-C. Lin, and K. S. Anseth, "A Microwell Cell Culture Platform for the Aggregation of Pancreatic $\beta$-Cells," Tissue Eng. Part C Methods, vol. 18, no. 8, pp. 583-592, Feb. 2012.

[21] A. Forget et al., "Rapid fabrication of functionalised poly(dimethylsiloxane) microwells for cell aggregate formation," Biomater. Sci., vol. 5, no. 4, pp. 828-836, 2017.

[22] Y. Y. Choi, B. G. Chung, D. H. Lee, A. Khademhosseini, J. H. Kim, and S. H. Lee, "Controlledsize embryoid body formation in concave microwell arrays," Biomaterials, vol. 31, no. 15, pp. 4296-4303, May 2010.

[23] Y. Ichihara, R. Utoh, M. Yamada, T. Shimizu, and Y. Uchigata, "Size effect of engineered islets prepared using microfabricated wells on islet cell function and arrangement," Heliyon, vol. 2, no. 6, 2016.

[24] O. Jeon, R. Marks, D. Wolfson, and E. Alsberg, "Dual-crosslinked hydrogel microwell system for formation and culture of multicellular human adipose tissue-derived stem cell spheroids," J. Mater. Chem. B, vol. 4, no. 20, pp. 3526-3533, 2016.

[25] T. Nishikawa et al., "Using size-controlled multicellular spheroids of murine adenocarcinoma cells to efficiently establish pulmonary tumors in mice," Biotechnol. J., vol. 12, no. 8, pp. 1-11, 2017.

[26] N. Udomluck, S. H. Kim, H. Cho, J. Y. Park, and H. Park, "Three-dimensional cartilage tissue regeneration system harnessing goblet-shaped microwells containing biocompatible hydrogel," Biofabrication, vol. 12, no. 1, p. 015019, 2019.

[27] S. E. Chung et al., "One-step pipetting and assembly of encoded chemical-laden microparticles for high-throughput multiplexed bioassays," Nat. Commun., vol. 5, pp. 112, 2014.

[28] J. J. Kim, K. W. Bong, E. Reátegui, D. Irimia, and P. S. Doyle, "Porous microwells for geometry-selective, large-scale microparticle arrays," Nat. Mater., vol. 16, no. 1, pp. 139146, 2017.

[29] J. R. Rettig and A. Folch, "Large-scale single-cell trapping and imaging using microwell arrays," Anal. Chem., vol. 77, no. 17, pp. 5628-5634, Sep. 2005. 
[30] J. Y. Park et al., "Single cell trapping in larger microwells capable of supporting cell spreading and proliferation," Microfluid. Nanofluidics, vol. 8, no. 2, pp. 263-268, Feb. 2010.

[31] A. Rosenthal, A. Macdonald, and J. Voldman, "Cell patterning chip for controlling the stem cell microenvironment," Biomaterials, vol. 28, no. 21, pp. 3208-3216, Jul. 2007.

[32] S. Gobaa, S. Hoehnel, M. Roccio, A. Negro, S. Kobel, and M. P. Lutolf, "Artificial niche microarrays for probing single stem cell fate in high throughput," Nat. Methods, vol. 8, no. 11, pp. 949-955, Nov. 2011.

[33] B. Xiong, K. Ren, Y. Shu, Y. Chen, B. Shen, and H. Wu, "Recent developments in microfluidics for cell studies," Adv. Mater., vol. 26, no. 31, pp. 5525-5532, Aug. 2014.

[34] C. P. Jen, J. H. Hsiao, and N. A. Maslov, "Single-cell chemical lysis on microfluidic chips with arrays of microwells," Sensors, vol. 12, no. 1, pp. 347-358, Jan. 2012.

[35] S. Lindström, K. Mori, T. Ohashi, and H. Andersson-Svahn, "A microwell array device with integrated microfluidic components for enhanced single-cell analysis," Electrophoresis, vol. 30, no. 24, pp. 4166-4171, Dec. 2009.

[36] L. Lin, Q. Chen, and J. Sun, "Micro/nanofluidics-enabled single-cell biochemical analysis," TrAC - Trends Anal. Chem., vol. 99, pp. 66-74, 2018.

[37] Q. Chen, J. Wu, Y. Zhang, Z. Lin, and J. M. Lin, "Targeted isolation and analysis of single tumor cells with aptamer-encoded microwell array on microfluidic device," Lab Chip, vol. 12, no. 24, pp. 5180-5185, Dec. 2012.

[38] M. C. Park, J. Y. Hur, H. S. Cho, S. H. Park, and K. Y. Suh, "High-throughput single-cell quantification using simple microwell-based cell docking and programmable time-course live-cell imaging," Lab Chip, vol. 11, no. 1, pp. 79-86, Jan. 2011.

[39] X. A. Figueroa, G. A. Cooksey, S. V. Votaw, L. F. Horowitz, and A. Folch, "Large-scale investigation of the olfactory receptor space using a microfluidic microwell array," Lab Chip, vol. 10, no. 9, pp. 1097-1212, 2010.

[40] J. A. James, M. Sushmitha, R. Premkumar, V. Narayanamurthy, and R. Kalpana, "Microfluidic micro-well (size and shape) by numerical optimization for single cell applications: Vertical trapping approach," in 2017 International Conference on Microelectronic Devices, Circuits and Systems, ICMDCS 2017, 2017, pp. 1-6.

[41] C. P. Jen, T. G. Amstislavskaya, Y. H. Liu, J. H. Hsiao, and Y. H. Chen, "Single-cell electric lysis on an electroosmotic-driven microfluidic chip with arrays of microwells," Sensors, vol. 12, no. 6, pp. 6967-6977, Jun. 2012.

[42] S. Lindström, M. Hammond, H. Brismar, H. Andersson-Svahn, and A. Ahmadian, "PCR amplification and genetic analysis in a microwell cell culturing chip," Lab Chip, vol. 9, no. 24, pp. 3465-3471, 2009.

[43] S. Bose et al., "Scalable microfluidics for single-cell RNA printing and sequencing," Genome Biol., vol. 16, no. 1, p. 20, Jun. 2015. 
[44] J. C. Love, J. L. Ronan, G. M. Grotenbreg, A. G. Van Der Veen, and H. L. Ploegh, “A microengraving method for rapid selection of single cells producing antigen-specific antibodies," Nat. Biotechnol., vol. 24, no. 6, pp. 703-707, Jun. 2006.

[45] S. Chao, M. R. Holl, S. C. McQuaide, T. T. H. Ren, S. A. Gales, and D. R. Meldrum, "Phosphorescence lifetime based oxygen micro-sensing using a digital micromirror device," Opt. Express, vol. 15, no. 17, p. 10681, 2007.

[46] T. W. Molter et al., "Algorithm advancements for the measurement of single cell oxygen consumption rates," in Proceedings of the 3rd IEEE International Conference on Automation Science and Engineering, IEEE CASE 2007, 2007, pp. 386-391.

[47] T. W. Molter et al., "A new approach for measuring single-cell oxygen consumption rates," IEEE Trans. Autom. Sci. Eng., vol. 5, no. 1, pp. 32-40, Jan. 2008.

[48] T. W. Molter et al., "A microwell array device capable of measuring single-cell oxygen consumption rates," Sensors Actuators, B Chem., vol. 135, no. 2, pp. 678-686, Jan. 2009.

[49] E. Ostuni, C. S. Chen, D. E. Ingber, and G. M. Whitesides, "Selective deposition of proteins and cells in arrays of microwells," Langmuir, vol. 17, no. 9, pp. 2828-2834, May 2001.

[50] A. Khademhosseini et al., "Co-culture of human embryonic stem cells with murine embryonic fibroblasts on microwell-patterned substrates," Biomaterials, vol. 27, no. 36, pp. 5968-5977, Dec. 2006.

[51] J. M. Karp et al., "Controlling size, shape and homogeneity of embryoid bodies using poly(ethylene glycol) microwells," Lab Chip, vol. 7, no. 6, pp. 786-794, 2007.

[52] H. C. Moeller, M. K. Mian, S. Shrivastava, B. G. Chung, and A. Khademhosseini, "A microwell array system for stem cell culture," Biomaterials, vol. 29, no. 6, pp. 752-763, Feb. 2008.

[53] Y.S. Hwang et al., "Microwell-mediated control of embryoid body size regulates embryonic stem cell fate via differential expression of WNT5a and WNT11," Proc. Natl. Acad. Sci., vol. 106, no. 40, pp. 16978-16983, Oct. 2009.

[54] J. M. Lee, J. E. Kim, E. Kang, S. H. Lee, and B. G. Chung, "An integrated microfluidic culture device to regulate endothelial cell differentiation from embryonic stem cells," Electrophoresis, vol. 32, no. 22, pp. 3133-3137, 2011.

[55] D. S. Decrop et al., "Single-Step Manufacturing of Femtoliter Microwell Arrays in a Novel Surface Energy Mimicking Polymer," in Transducers, 2015, pp. 514-517.

[56] J. Lee, P. W. Bisso, R. L. Srinivas, J. J. Kim, A. J. Swiston, and P. S. Doyle, "Universal processinert encoding architecture for polymer microparticles," Nat. Mater., vol. 13, no. 5, pp. 524-529, 2014.

[57] S. Han et al., "Lithographically encoded polymer microtaggant using high-capacity and error-correctable QR Code for anti-counterfeiting of drugs," Adv. Mater., vol. 24, no. 44, pp. 5924-5929, Nov. 2012.

[58] A. A. S. Bhagat, H. Bow, H. W. Hou, S. J. Tan, J. Han, and C. T. Lim, "Microfluidics for cell separation," Medical and Biological Engineering and Computing, vol. 48, no. 10. pp. 999- 
1014, Oct-2010.

[59] I. Inoue, Y. Wakamoto, H. Moriguchi, K. Okano, and K. Yasuda, “On-chip culture system for observation of isolated individual cells," Lab Chip, vol. 1, no. 1, pp. 50-55, Sep. 2001.

[60] S. H. Kim, S. Iwai, S. Araki, S. Sakakihara, R. lino, and H. Noji, "Large-scale femtoliter droplet array for digital counting of single biomolecules," Lab Chip, vol. 12, no. 23, pp. 4986-4991, Dec. 2012.

[61] A. Hattori, T. Yagi, and C. W. Tsao, "Encapsulation of single cells into fixed droplets using triangular microwells," Electron. Commun. Japan, vol. 99, no. 2, pp. 55-63, Feb. 2016.

[62] C. H. Lin et al., "A microfluidic dual-well device for high-throughput single-cell capture and culture," Lab Chip, vol. 15, no. 14, pp. 2928-2938, Jul. 2015.

[63] A. Khademhosseini et al., "Cell docking inside microwells within reversibly sealed microfluidic channels for fabricating multiphenotype cell arrays," Lab Chip, vol. 5, no. 12, pp. 1380-1386, 2005.

[64] Y. Tokimitsu et al., "Single lymphocyte analysis with a microwell array chip," Cytom. Part A, vol. 71, no. 12, pp. 1003-1010, Dec. 2007.

[65] M. Ochsner et al., "Micro-well arrays for 3D shape control and high resolution analysis of single cells," Lab Chip, vol. 7, no. 8, pp. 1074-1077, 2007.

[66] M. Deutsch et al., "A novel miniature cell retainer for correlative high-content analysis of individual untethered non-adherent cells," Lab Chip, vol. 6, no. 8, pp. 995-1000, 2006.

[67] C. C. Meek and P. Pantano, "Spatial confinement of avidin domains in microwell arrays," Lab Chip, vol. 1, no. 2, pp. 158-163, Dec. 2001.

[68] Q. Pan and A. E. Herr, "Geometry-induced injection dispersion in single-cell protein electrophoresis," Anal. Chim. Acta, vol. 1000, pp. 214-222, Feb. 2018.

[69] C. M. Nelson, S. Raghavan, J. L. Tan, and C. S. Chen, "Degradation of micropatterned surfaces by cell-dependent and -independent processes," Langmuir, vol. 19, no. 5, pp. 1493-1499, 2003.

[70] A. J. You, R. J. Jackman, G. M. Whitesides, and S. L. Schreiberl, "A miniaturized ariayed assay format for detecting small molecule-protein interactions in cells," Chem. Biol., vol. 4, no. 12, pp. 969-975, 1997.

[71] V. I. Chin, P. Taupin, S. Sanga, J. Scheel, F. H. Gage, and S. N. Bhatia, "Microfabricated platform for studying stem cell fates," Biotechnol. Bioeng., vol. 88, no. 3, pp. 399-415, Nov. 2004.

[72] S. Lindström, R. Larsson, and H. A. Svahn, "Towards high-throughput single cell/clone cultivation and analysis," Electrophoresis, vol. 29, no. 6, pp. 1219-1227, Mar. 2008.

[73] A. Revzin, R. G. Tompkins, and M. Toner, "Surface engineering with poly(ethylene glycol) photolithography to create high-density cell arrays on glass," Langmuir, vol. 19, no. 23, pp. 9855-9862, Nov. 2003. 
[74] J. M. Cha et al., "Embryoid body size-mediated differential endodermal and mesodermal differentiation using polyethylene glycol (PEG) microwell array," Macromol. Res., vol. 23, no. 3, pp. 245-255, Mar. 2015.

[75] H. Tekin, M. Anaya, M. D. Brigham, C. Nauman, R. Langer, and A. Khademhosseini, "Stimuliresponsive microwells for formation and retrieval of cell aggregates," Lab Chip, vol. 10, no. 18, pp. 2411-2418, Sep. 2010.

[76] H. H. Jeong, J. H. Lee, Y. M. Noh, Y. G. Kim, and C. S. Lee, "Generation of uniform agarose microwells for cell patterning by micromolding in capillaries," Macromol. Res., vol. 21, no. 5, pp. 534-540, 2013.

[77] L. C. Taylor and D. R. Walt, "Application of high-density optical microwell arrays in a livecell biosensing system," Anal. Biochem., vol. 278, no. 2, pp. 132-142, 2000.

[78] Y. Kuang, I. Biran, and D. R. Walt, "Living bacterial cell array for genotoxin monitoring," Anal. Chem., vol. 76, no. 10, pp. 2902-2909, May 2004.

[79] M. Nikkhah, J. S. Strobl, V. Srinivasaraghavan, and M. Agah, "Isotropically etched silicon microarrays for rapid breast cancer cell capture," IEEE Sens. J., vol. 13, no. 3, pp. 11251132, 2013.

[80] P. Zellner, L. Renaghan, Z. Hasnain, and M. Agah, "A fabrication technology for threedimensional micro total analysis systems," J. Micromechanics Microengineering, vol. 20, no. 4, 2010.

[81] S. Yamamura et al., "Single-cell microarray for analyzing cellular response," Anal. Chem., vol. 77, no. 24, pp. 8050-8056, Dec. 2005.

[82] Š. Selimović, F. Piraino, H. Bae, M. Rasponi, A. Redaelli, and A. Khademhosseini, "Microfabricated polyester conical microwells for cell culture applications," Lab Chip, vol. 11, no. 14, pp. 2325-2332, Jul. 2011.

[83] C. Gregory and M. Veeman, "3D-printed microwell arrays for Ciona microinjection and timelapse imaging," PLoS One, vol. 8, no. 12, Dec. 2013.

[84] N. Xue, L. Bleris, and J.-B. (JB) Lee, "One-step fabrication of three-dimensional polydimethylsiloxane peel-off microwell array for cell trapping," J. Micro/Nanolithography, MEMS, MOEMS, vol. 14, no. 1, p. 014503, Feb. 2015.

[85] J. Park, M. Müller, J. Kim, and H. Seidel, "Fabrication of a cell-adhesive microwell array for 3-dimensional in vitro cell model," Biomed. Eng. Lett., vol. 5, no. 2, pp. 140-146, Jun. 2015.

[86] M. Kawai et al., "Single-cell Trapping Using Microwell Arrays Fabricated from Selfassembled Particle Monolayers," Mol. Cryst. Liq. Cryst., vol. 603, no. 1, pp. 248-255, Nov. 2014.

[87] G. H. Lee, Y. E. Park, M. Cho, H. Park, and J. Y. Park, "Magnetic force-assisted self-locking metallic bead array for fabrication of diverse concave microwell geometries," Lab Chip, vol. 16, no. 18, pp. 3565-3575, 2016.

[88] A. R. Thomsen et al., "A deep conical agarose microwell array for adhesion independent 
three-dimensional cell culture and dynamic volume measurement," Lab Chip, vol. 18, no. 1, pp. 179-189, 2017.

[89] L. Kang, M. J. Hancock, M. D. Brigham, and A. Khademhosseini, "Cell confinement in patterned nanoliter droplets in a microwell array by wiping," J. Biomed. Mater. Res. - Part $A$, vol. 93, no. 2, pp. 547-557, May 2010.

[90] J. J. Kim, K. W. K. W. Bong, E. Reátegui, D. Irimia, and P. S. Doyle, "Porous microwells for geometry-selective, Large-scale microparticle arrays," in 20th International Conference on Miniaturized Systems for Chemistry and Life Sciences, MicroTAS 2016, 2016, vol. 16, no. January, pp. 1085-1086.

[91] Y. Wang, P. Shah, C. Phillips, C. E. Sims, and N. L. Allbritton, "Trapping cells on a stretchable microwell array for single-cell analysis," Anal. Bioanal. Chem., vol. 402, no. 3, pp. 10651072, 2012.

[92] A. Revzin, K. Sekine, A. Sin, R. G. Tompkins, and M. Toner, "Development of a microfabricated cytometry platform for characterization and sorting of individual leukocytes," Lab Chip, vol. 5, no. 1, pp. 30-37, 2005.

[93] R. J. Jackman, D. C. Duffy, E. Ostuni, N. D. Willmore, and G. M. Whitesides, "Fabricating Large Arrays of Microwells with Arbitrary Dimensions and Filling Them Using Discontinuous Dewetting," Anal. Chem., vol. 70, no. 11, pp. 2280-2287, Jun. 1998.

[94] I. Biran and D. R. Walt, "Optical imaging fiber-based single live cell arrays: A high-density cell assay platform," Anal. Chem., vol. 74, no. 13, pp. 3046-3054, Jul. 2002.

[95] B. Cordovez, D. Psaltis, and D. Erickson, "Trapping and storage of particles in electroactive microwells," Appl. Phys. Lett., vol. 90, no. 2, 2007.

[96] S. H. Kim, T. Yamamoto, D. Fourmy, and T. Fujii, "Electroactive microwell arrays for highly efficient single-cell trapping and analysis," Small, vol. 7, no. 22, pp. 3239-3247, 2011.

[97] M. C. Park, J. Y. Hur, K. W. Kwon, S. H. Park, and K. Y. Suh, "Pumpless, selective docking of yeast cells inside a microfluidic channel induced by receding meniscus," Lab Chip, vol. 6, no. 8, pp. 988-994, 2006.

[98] P. Shah, X. Zhu, C. Chen, Y. Hu, and C. Z. Li, "Lab-on-chip device for single cell trapping and analysis," Biomed. Microdevices, vol. 16, no. 1, pp. 35-41, Feb. 2014.

[99] D. Dendukuri, P. Panda, R. Haghgooie, J. M. Kim, T. A. Hatton, and P. S. Doyle, "Modeling of oxygen-inhibited free radical photopolymerization in a PDMS microfluidic device," Macromolecules, vol. 41, no. 22, pp. 8547-8556, 2008.

[100] J. Li, Y. Osada, and J. Cooper-White, Functional Hydrogels As Biomaterials. Springer, 2018.

[101] D. Dendukuri, S. S. Gu, D. C. Pregibon, T. A. Hatton, and P. S. Doyle, "Stop-flow lithography in a microfluidic device," Lab Chip, vol. 7, no. 7, pp. 818-828, 2007.

[102] M. Li, M. Humayun, J. A. Kozinski, and D. K. Hwang, "Functional polymer sheet patterning using microfluidics," Langmuir, vol. 30, no. 28, pp. 8637-8644, 2014. 
[103] D. K. Hwang et al., "Stop-Flow Lithography for the Production of Shape-Evolving Degradeable Microgel Particles," vol. 131, no. 12, pp. 4499-4504, 2009.

[104] N. Hakimi, S. S. H. Tsai, C. H. Cheng, and D. K. Hwang, "One-step two-dimensional microfluidics-based synthesis of three-dimensional particles," Adv. Mater., vol. 26, no. 9, pp. 1393-1398, 2014.

[105] Y. Ito et al., "The movement of a water droplet on a gradient surface prepared by photodegradation," Langmuir, vol. 23, no. 4, pp. 1845-1850, 2007. 